\title{
The self-linking number in annulus and pants open book decompositions
}

\author{
KEIKO KaWAMURO \\ Elena PAVELESCU
}

\begin{abstract}
We find a self-linking number formula for a given null-homologous transverse link in a contact manifold that is compatible with either an annulus or a pair of pants open book decomposition. It extends Bennequin's self-linking formula for a braid in the standard contact 3-sphere.
\end{abstract}

57M25, 57M27; 57M50

\section{Introduction}

Alexander's theorem [1] states that every closed and oriented 3-manifold admits an open book decomposition.

Definition 1.1 Let $\Sigma$ be a surface with nonempty boundary and $\phi$ be a diffeomorphism of the surface fixing the boundary pointwise. We construct a closed manifold

$$
M_{(\Sigma, \phi)}=\Sigma \times[0,1] / \sim
$$

where " $\sim$ " is an equivalence relation satisfying $(\phi(x), 0) \sim(x, 1)$ for $x \in \operatorname{Int}(\Sigma)$ and $(x, \tau) \sim(x, 1)$ for $x \in \partial \Sigma$ and $\tau \in[0,1]$. The pair $(\Sigma, \phi)$ is called an abstract open book decomposition of the manifold $M_{(\Sigma, \phi)}$.

Alternatively, an open book decomposition for $M$ can be defined as a pair $(L, \pi)$, where (1) $L$ is an oriented link in $M$ called the binding of the open book; (2) $\pi: M \backslash L \rightarrow S^{1}$ is a fibration whose fiber, $\pi^{-1}(\theta)$, called a page, is the interior of a compact surface $\Sigma_{\theta} \subset M$ such that $\partial \Sigma_{\theta}=L$ for all $\theta \in S^{1}$.

One of the central results about the topology of contact 3-manifolds is "the Giroux correspondence" [11]:

$$
\left\{\begin{array}{l}
\text { contact structures } \xi \text { on } M^{3} \\
\text { up to contact isotopy }
\end{array}\right\} \stackrel{1-1}{\longleftrightarrow}\left\{\begin{array}{l}
\text { open book decompositions }(\Sigma, \phi) \\
\text { of } M^{3} \text { up to positive stabilization }
\end{array}\right\}
$$


For example, the standard contact structure $\xi_{\text {std }}=\operatorname{ker}\left(d z+r^{2} d \theta\right)$ on $S^{3}=\mathbb{R}^{3} \cup\{\infty\}$ corresponds to the open book decomposition $\left(D^{2}\right.$, id $)$.

We define a braid and the braid index in a general open book setting:

Definition 1.2 Suppose $(L, \pi)$ is an open book decomposition for a 3-manifold $M$. A link $K \subset M$ is called a (closed) braid if $K$ transversely intersects each page $\Sigma_{\theta}=$ $\pi^{-1}(\theta)$ of the open book. That is, at each point $p \in K \cap \Sigma_{\theta}$, we have $T_{p} \Sigma_{\theta} \oplus T_{p} K=$ $T_{p} M$. The braid index of a braid $K$ is the degree of the map $\pi$ restricted to $K$. In other words, if a braid $K$ intersects each page in $n$ points, then the braid index of $K$ is $n$.

Bennequin [2] proved that any transverse link in $\left(S^{3}, \xi_{\text {std }}\right)$ can be transversely isotoped to a closed braid in $\left(D^{2}\right.$,id). Later the second author generalized Bennequin's result into the following:

Theorem 1.3 [15, Theorem 3.2.1] Suppose $(\Sigma, \phi)$ is an open book decomposition for a 3-manifold $M=M_{(\Sigma, \phi)}$. Let $\xi=\xi_{(\Sigma, \phi)}$ be a compatible contact structure. Let $K$ be a transverse link in $(M, \xi)$. Then $K$ can be transversely isotoped to a braid in $(\Sigma, \phi)$.

The self-linking (Bennequin) number is a classical invariant for transverse knots. Bennequin [2] gave a formula of the self-linking number for a braid $b$ in $\left(D^{2}\right.$, id):

$$
\operatorname{sl}(b)=-n+a,
$$

where $n$ is the braid index and $a$ the algebraic crossing number (the exponent sum) of the braid.

The first goal of this paper is to give a combinatorial description for the self-linking number of a null-homologous transverse link in the contact lens spaces compatible with $\left(A, D^{k}\right)$, the annulus $A$ open book decomposition with monodromy the $k$-th power of the positive Dehn twist $D$. By Theorem 1.3, our problem is reduced to searching for a self-linking formula for a null-homologous braid in the open book decomposition $\left(A, D^{k}\right)$. Such a braid is given by a product of permutations of points in a local disk on the annulus $A$ and moves of points which turn around the hole of $A$. We denote by $a_{\sigma}$ the algebraic crossing number of the local permutations, and by $a_{\rho}$ the algebraic rotation number around the hole of $A$; see Definition 2.5 for precise definitions. With this notation, we extend Bennequin's formula (1-1) to the following: 
Theorem 4.1 Let $b$ be a null-homologous closed braid in $\left(A, D^{k}\right)$ of braid index $n$. For $k \neq 0$ we have

$$
\operatorname{sl}(b)=-n+a_{\sigma}+a_{\rho}\left(1-\frac{a_{\rho}}{k}\right) .
$$

When $k=0$ there exists a canonical Seifert surface $\Sigma_{b}$ of $b$ and we have

$$
\operatorname{sl}\left(b, \Sigma_{b}\right)=-n+a_{\sigma} .
$$

The Seifert surface $\Sigma_{b}$ will be constructed in Section 3. The surface is canonical in the sense that the way of construction is similar to that of the standard Seifert surface, or Bennequin surface, of a closed braid in $S^{3}$.

Our second goal is to find a self-linking formula for null-homologous transverse links in a contact Seifert fibered manifold $M$ of signature $\left(g=0, k_{1}, k_{2}, k_{3}\right)$. Let $S$ be a pair of pants (a disk with two holes). Let $D_{i}(i=1,2,3)$ be the positive Dehn twists along the curves parallel to the boundary circles of $S$. Then $M$ has an open book decomposition $\left(S, D_{1}^{k_{1}} \circ D_{2}^{k_{2}} \circ D_{3}^{k_{3}}\right)$, and is equipped with a compatible contact structure. A braid in the pants open book is a product of permutations of points in a local disk on $S$ and moves of points which turn around the holes of $S$. We denote by $a_{\sigma}$ the algebraic crossing number of the local permutations and by $a_{\rho_{i}}(i=2,3)$ the algebraic winding number around the holes. See Definition 5.4 for precise definitions. We obtain the following formula which also extends (1-1).

Theorem 5.6 Let $b$ be a null-homologous braid in $\left(S, D_{1}^{k_{1}} \circ D_{2}^{k_{2}} \circ D_{3}^{k_{3}}\right)$ of braid index $n$. We have

$$
\operatorname{sl}\left(b,\left[\Sigma_{b}\right]\right)=-n+a_{\sigma}+a_{\rho_{2}}\left(1-s_{2}\right)+a_{\rho_{3}}\left(1-s_{3}\right)-\left(s_{2}+s_{3}\right) k_{1},
$$

where $\Sigma_{b}$ is some Seifert surface for $b$. The constants $s_{2}, s_{3}$ are determined by $a_{\rho_{2}}, a_{\rho_{3}}, k_{1}, k_{2}$ and $k_{3}$, under the assumption that $b$ is null-homologous; see Definition 5.4.

The organization of the paper is the following:

In Section 2, we fix notation and study properties of the contact lens space $\left(M_{\left(A, D^{k}\right)}, \xi_{k}\right)$. In Section 3, we construct a Bennequin type Seifert surface $\widehat{F}_{b}$ for a given braid $b$ in $\left(A, D^{k}\right)$. In general, this $\widehat{F}_{b}$ is an immersed surface and the Bennequin-Eliashberg inequality is not satisfied even for tight cases. We resolve all the singularities and obtain an embedded surface $\Sigma_{b}$. We develop a theory about resolution of singularities of an immersed surface and corresponding changes in characteristic foliations.

In Section 4, we prove Theorem 4.1, an explicit formula of the self-linking number relative to $\Sigma_{b}$, which extends Bennequin's formula (1-1). As the self-linking number 
is defined to be the Euler number of the contact 2-plane bundle relative to the surface framing, we measure the difference between the immersed $\widehat{F}_{b}$-framing and the embedded $\Sigma_{b}$-framing. We also study the behavior of our self-linking number under a braid stabilization. Corollary 4.5 states that our self-linking number is invariant under a positive stabilization and changes by 2 under a negative stabilization, which extends Bennequin's result for braids in $\left(S^{3}, \xi_{\text {std }}\right)$.

In Section 5, we apply our surface construction method to some class of contact Seifert fibered manifolds and prove Theorem 5.6.

Acknowledgements The authors would like to thank John Etnyre for numerous useful comments and sharing his ideas, especially those on Corollary 3.9, and Matthew Hedden for helpful comments on Section 4. They also thank the referee for carefully examining the paper and providing constructive comments. KK thanks Tim Cochran and Walter Neumann for stimulating conversations. She was partially supported by NSF grants DMS-0806492 and DMS-0635607.

\section{Preliminaries}

Let $A=S^{1} \times I$ be an annulus and $D_{\alpha}$ the positive Dehn twist about the core circle $\alpha=S^{1} \times\{1 / 2\}$. For simplicity, we denote $D_{\alpha}$ by $D$.

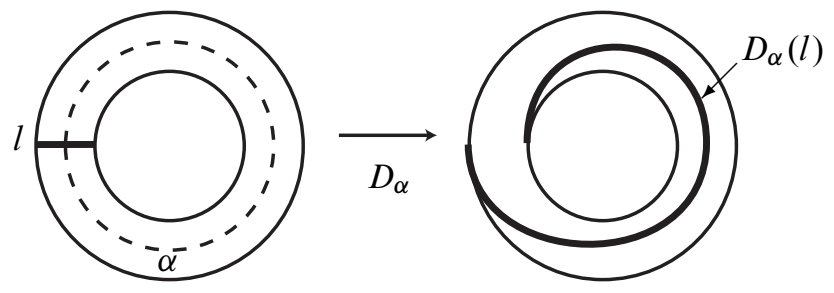

Figure 1: A positive Dehn twist $D_{\alpha}$ about $\alpha$

We study an abstract open book decomposition $\left(A, D^{k}\right)$.

Claim 2.1 The corresponding manifold $M_{\left(A, D^{k}\right)}$ to $\left(A, D^{k}\right)$ is

$$
M_{\left(A, D^{k}\right)}= \begin{cases}L(k, k-1) & \text { if } k>0 \\ S^{1} \times S^{2} & \text { if } k=0 \\ L(|k|, 1) & \text { if } k<0\end{cases}
$$


Proof Let $D_{\circ} \simeq D^{2}$ be a disk and $\gamma:=\partial D_{\circ}$. Recall that $\left(D_{\circ}\right.$, id $)$ is a planar open book decomposition for $\left(S^{3}, \xi_{\text {std }}\right)$. Let $D_{\mu} \subset D_{\circ}$ be a disc with boundary $\mu$. The core of the solid torus $D_{\mu} \times S^{1} \subset S^{3}$ is the unknot, $U$. The meridian of the torus $T_{\mu}=\partial\left(D_{\mu} \times S^{1}\right)$ is $\mu$. Pick a point $p \in \mu$, and define a longitude $\lambda$ of $T_{\mu}$ as $\lambda=\{p\} \times S^{1}$. Remove $D_{\mu} \times S^{1}$ from $S^{3}$, and attach a new solid torus by identifying its meridian $m$ with $\lambda$ and its longitude $l$ with $-\mu$. This is the 0 -surgery along the unknot $U$. The resulting manifold is $S^{1} \times S^{2}$. In this way we get an open book decomposition $\left(A, \mathrm{id}_{A}\right)$ for $S^{1} \times S^{2}$, whose page $A$ is the union of the annulus $D_{\circ} \backslash D_{\mu}$, shaded in Figure 2 (1), and the annulus bounded by $-l$ and the core $\gamma^{\prime}$ of the solid torus, sketched in Figure 2 (2).

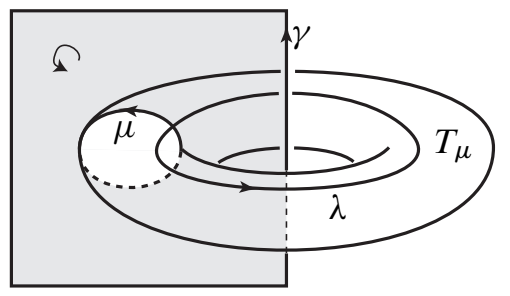

(2)

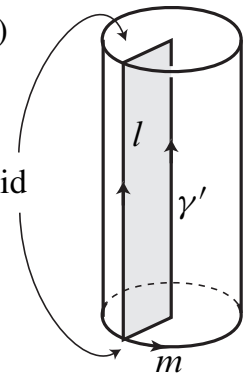

(3)

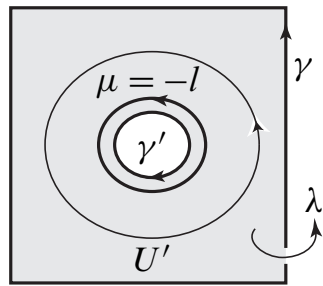

Figure 2: (1) Removing a solid torus $D_{\mu} \times S^{1}$ from $S^{3} \quad$ (2) The attaching solid torus (3) The page annulus $A$

The Dehn twist $D^{k}$ about the core $U^{\prime} \subset\left(D_{\circ} \backslash D_{\mu}\right) \subset A$, sketched in Figure 2 (3), of the page annulus $A$ is equivalent to applying $(1 /-k)$-surgery along the unknot $U^{\prime}$. The link $\left(U \cup U^{\prime}\right) \subset S^{3}$ is the positive Hopf link. By the slam-dunk operation, the surgery description is reduced to the $k$-surgery along $U$, which represents $L(k,-1)=$ $L(k, k-1)$ when $k>0$ and $L(|k|, 1)$ when $k<0$.

Let $\left(M_{\left(A, D^{k}\right)}, \xi_{k}\right)$ be the contact manifold corresponds to the open book $\left(A, D^{k}\right)$.

Claim 2.2 The contact manifold $\left(M_{\left(A, D^{k}\right)}, \xi_{k}\right)$ is overtwisted if and only if $k<0$. When $k \geq 0$, this $\xi_{k}$ is the unique tight contact structure for $L(k, k-1)$.

Proof If $k<0$, Goodman's criterion for overtwistedness [12, Theorem 1.2] implies that $\xi_{k}$ is overtwisted.

When $k=0$, according to Etnyre and Honda [7, Proof of Lemma 3.2], the open book is a boundary of a positive Lefschetz fibration on a 4-manifold $X$, so that $\left(S^{1} \times S^{2}, \xi_{0}\right)$ is Stein filled by $X$, hence tight. Moreover, $\xi_{0}$ is the unique tight contact structure on $S^{2} \times S^{1}$ due to Eliashberg [4]. 
When $k>0$, the monodromy is a product of positive Dehn twists. Etnyre and Honda's [7, Lemma 3.2] guarantees that the contact structure compatible with such an open book is Stein fillable, hence tight. The uniqueness for $k>0$ follows from Honda's classification of tight contact structures for lens spaces [13]. More precisely, we have

$$
-\frac{k}{k-1}=-2-\frac{1}{-2-\frac{1}{-2-\cdots-\frac{1}{-2}}}=[-2,-2, \ldots,-2] \text {, repeating }(k-1) \text { times }
$$

and $|(-2+1)(-2+1) \cdots(-2+1)|=1$, thus the manifold has the unique tight contact structure.

We fix notation. See Figure 3. Suppose we have a null-homologous closed braid $b$ of braid index $n$ in the open book $\left(A, D^{k}\right)$. Let $\gamma \cup \gamma^{\prime}=\partial A$ whose orientations are induced by that of $A$. Let $A_{\theta}(\theta \in[0,1])$ denote the page $A \times\{\theta\} \subset M_{\left(A, D^{k}\right)}$. Under the identification $A=S^{1} \times[0,1]$, we set $\alpha=S^{1} \times\{1 / 2\}$. Let $\beta$ be a circle between $\alpha$ and $\gamma$ which is oriented clockwise.

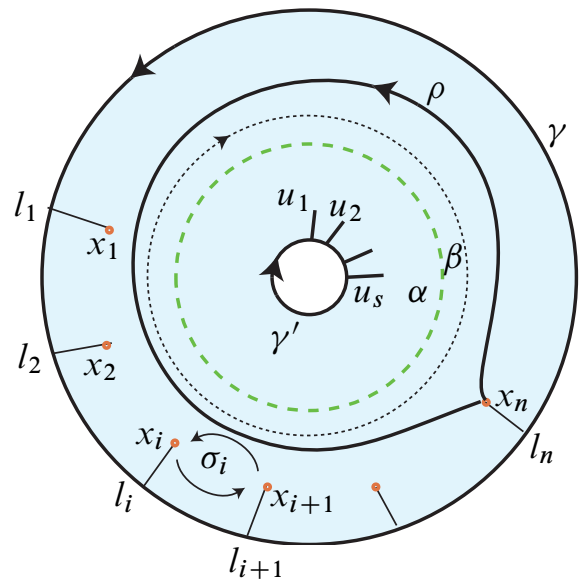

Figure 3

Assumption 2.3 Choose points $x_{1}, \ldots, x_{n}$ sitting between $\gamma$ and $\alpha$. By braid isotopy, which preserves the transverse knot class (see Theorem 2.8 (2)), we may assume that

$$
b \cap A_{0}=\left\{x_{1}, \ldots, x_{n}\right\} .
$$

Let $\sigma_{i}(i=1, \ldots, n-1)$ be the generators of Artin's braid group $B_{n}$ satisfying $\sigma_{i} \sigma_{i+1} \sigma_{i}=\sigma_{i+1} \sigma_{i} \sigma_{i+1}$ and $\sigma_{i} \sigma_{j}=\sigma_{j} \sigma_{i}$ for $|i-j| \geq 2$. Geometrically, $\sigma_{i}$ acts by switching the marked points $x_{i}$ and $x_{i+1}$ counterclockwise. The circle $\beta$ will appear 
in Section 3.1. Let $\rho$ be a braid element which moves $x_{n}$ once around the annulus in the indicated direction.

Proposition 2.4 An $n$-strand braid $b$ in $\left(A, D^{k}\right)$ has a braid word in $\left\{\sigma_{1}, \ldots, \sigma_{n-1}, \rho\right\}$.

Proof Let $A^{*}$ be the annulus with $n$-marked points $x_{1}, \ldots, x_{n}$. Let $C(A, n)$ denote the configuration space of $n$ distinct unordered points in $A$. The fundamental group $\pi_{1}(C(A, n))$ is the $n$-stranded surface braid group of $A$. Let $\operatorname{Mod}(A)$ be the mapping class group of annulus $A$ fixing the boundaries pointwise. Recall the generalized Birman exact sequence [3; 9, Theorem 9.1]:

$$
1 \longrightarrow \pi_{1}(C(A, n)) \stackrel{\text { Push }}{\longrightarrow} \operatorname{Mod}\left(A^{*}\right) \stackrel{\text { Forget }}{\longrightarrow} \operatorname{Mod}(A) \longrightarrow 1
$$

Since the map $\mathcal{F}$ orget is forgetting the $n$ marked points, $\operatorname{ker}(\mathcal{F}$ orget $)=\pi_{1}(C(A, n))$ is generated by $\left\{\sigma_{1}, \ldots, \sigma_{n-1}, \rho\right\}$.

Definition 2.5 Let $a_{\sigma} \in \mathbb{Z}$ (resp. $a_{\rho} \in \mathbb{Z}$ ) be the exponent sum of $\sigma_{1}, \ldots, \sigma_{n-1}$ (resp. $\rho$ ) in the braid word of $b$.

Proposition 2.6 If $k>0$ (resp. $k<0$ ), we may assume that $a_{\rho} \geq 0$ (resp. $a_{\rho} \leq 0$ ).

To prove Proposition 2.6, we first define braid stabilization and recall its properties.

Definition 2.7 Let $b$ be a closed braid in an open book $(\Sigma, \phi)$. Suppose that $\lambda \subset \partial \Sigma$ is one of the bindings of the open book and $p \in\left(\Sigma_{\theta} \cap b\right)$ is a point; see Figure 4. Join $p$ and a point on $\lambda$ by an arc $a \subset\left(\Sigma_{\theta} \backslash b\right)$. A positive (negative) stabilization of $b$ about $\lambda$ along $a$ is pulling a small neighborhood of $p$ of the braid, then adding a positive (negative) kink about $\lambda$ in a neighborhood of $a$.
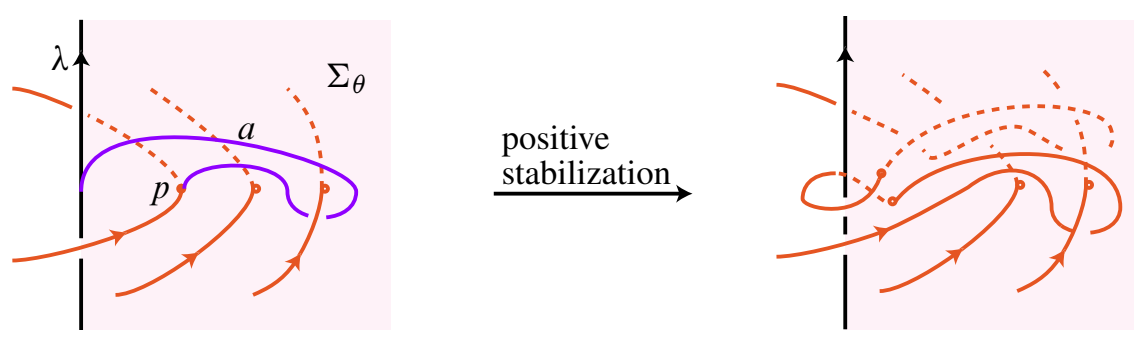

Figure 4: Positive braid stabilization along $a$

The second author proved Markov theorem in a general open book setting: 
Theorem 2.8 [15, Theorems 4.1.3 and 4.1.4]

(1) Two closed braids $K_{1}$ and $K_{2}$ in an open book decomposition have the same topological type if and only if they are related by braid isotopy, positive and negative braid stabilizations.

(2) The above $K_{1}, K_{2}$ are transversely isotopic if and only if they are related by braid isotopy and positive braid stabilizations.

Proof of Proposition 2.6 Suppose $b$ is an $n$-strand braid. Recall that $\left(A, D^{k}\right)$ has two binding components, $\gamma$ and $\gamma^{\prime}$. Let $a$ be an arc joining $x_{n}$ and $\gamma^{\prime}$ and intersecting $\alpha$ at a point as sketched in Figure 5. Pick a small line segment of the

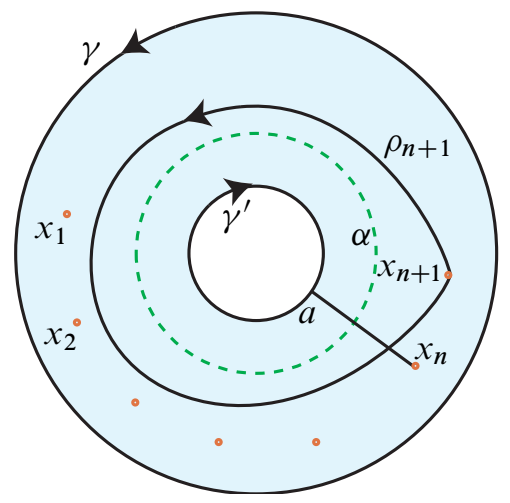

Figure 5: Definitions of $a, x_{n+1}$ and $\rho_{n+1}$

$n$-th strand in $A \times(1-\epsilon, 1)$, near the top page $A_{\theta=1}$ of the open book, and positively stabilize it along $a$. As a consequence, it gains a new braid strand, which we call $v$, lying in a small tubular neighborhood of $\gamma^{\prime}$; see Figure 6 (1). Put a point $x_{n+1} \subset A$ on the right side of $x_{n}$ between $\gamma$ and define $\rho_{n+1}$ a braid generator as in Figure 5 . Move $v$ by a braid isotopy supported in $A \times(1-\epsilon, 1+\epsilon)$ so that $v$ intersects the page $A_{0}=A_{1}$ at $x_{n+1}$. This isotopy introduces $\left(\rho_{n+1}\right)^{k}$ in $A \times(0, \epsilon)$ as a consequence of the monodromy $D^{k}$. Compare Figure 6 (1) and (2).

We observe that in a stabilized braid, $\rho_{n+1}$ plays the role of the old $\rho=\rho_{n}$ and as Figure 6 (3) shows, they are related by

$$
\rho_{n}=\sigma_{n} \rho_{n+1} \sigma_{n}
$$

Thus a positive stabilization about $\gamma^{\prime}$ takes a word $b$ to $\left(\rho_{n+1}\right)^{k} \tilde{b} \sigma_{n}$, where $\tilde{b}$ is obtained from $b$ replacing each $\rho$ with $\sigma_{n} \rho_{n+1} \sigma_{n}$. The data change in the following way:

$$
n \rightarrow n+1, \quad a_{\sigma} \rightarrow a_{\sigma}+1+2 a_{\rho}, \quad a_{\rho} \rightarrow a_{\rho}+k .
$$



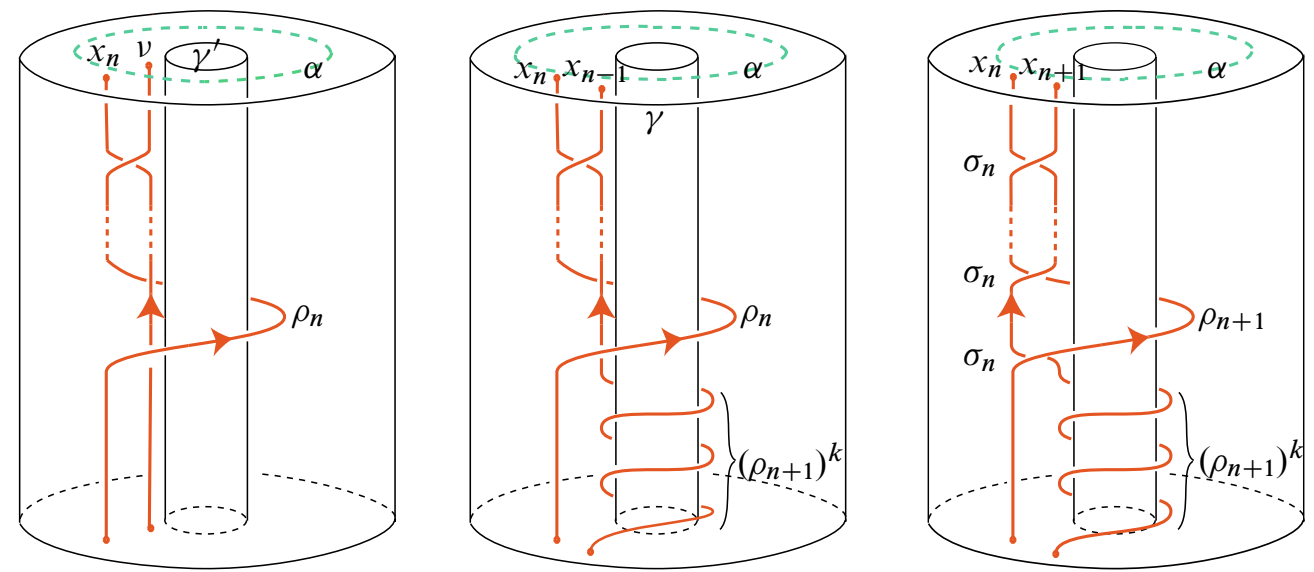

Figure 6: (1) Positive stabilization about the binding $\gamma^{\prime}$ (2) Transversely isotope $v$ near $\gamma^{\prime}$ to $x_{n+1}$ near $\gamma$. This introduces $k$ additional $\rho_{n+1}$ 's. (3) $\rho_{n}$ and $\rho_{n+1}$ are related by $\rho_{n}=\sigma_{n} \rho_{n+1} \sigma_{n}$.

Theorem 2.8 (2) tells that a positive stabilization preserves the transverse knot type, so if $k>0$ (resp. $k<0$ ) we may assume that $a_{\rho} \geq 0$ (resp. $a_{\rho} \leq 0$ ).

The next corollary introduces a number $s$ :

Corollary 2.9 If $k \neq 0$ there exists a nonnegative integer $s$ such that $a_{\rho}=s k$. If $k=0$ then $a_{\rho}=0$.

Proof In the homology group $H_{1}\left(M_{\left(A, D^{k}\right)} ; \mathbb{Z}\right)$, we have $[b]+a_{\rho}[\beta]=0$. Since the braid $b$ is null-homologous $a_{\rho}[\beta]=[b]=0$. The meridian $\mu$ introduced in the proof of Claim 2.1 is a generator of $H_{1}\left(M_{\left(A, D^{k}\right)} ; \mathbb{Z}\right)=\mathbb{Z} / k \mathbb{Z}$. Since $a_{\rho}[-\mu]=a_{\rho}[\beta]=0$ we have $a_{\rho} \equiv 0(\bmod k)$, implying the existence of $s \in \mathbb{Z}$ with $a_{\rho}=s k$ for $k \neq 0$. Proposition 2.6 guarantees that we may assume $s \geq 0$. When $k=0$, we have $a_{\rho}=0$.

\section{Construction of Seifert surface $\Sigma_{b}$}

The goal of this section is to construct a Seifert surface $\Sigma_{b}$ for a null-homologous braid $b$ whose braid word is written in $\left\{\sigma_{1}, \ldots, \sigma_{n-1}, \rho\right\}$. (By abuse of notation, we use $b$ for both the closed braid and its braid word.) We first construct a surface $F_{b}$ and change it to $\widetilde{F}_{b}$. We further deform $\widetilde{F}_{b} \rightarrow \check{F}_{b} \rightarrow \widehat{F}_{b}$ and finally obtain $\Sigma_{b}$. 


\subsection{Construction of the surface $F_{b}$}

Let $l_{i} \subset A$ be a line segment perpendicular to $\gamma$ having $x_{i}$ as one of its endpoints and with the other end on $\gamma$; see Figure 3. Since $l_{i}$ is disjoint from the Dehn twist curve $\alpha$, in the resulting manifold, $M_{\left(A, D^{k}\right)}$, the $\operatorname{arc} l_{i}$ swipes a disk $\delta_{i}:=\left(l_{i} \times[0,1]\right) / \sim$. See Figure 7. The center of $\delta_{i}$ is $l_{i} \cap \gamma$. We orient $\delta_{i}$ so that the binding $\gamma$ is positively transverse to $\delta_{i}$.

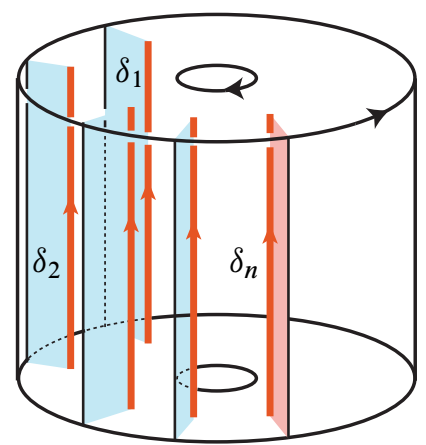

Figure 7: Oriented disks $\delta_{1}, \ldots, \delta_{n}$. The positive (negative) side is light blue (dark pink).

If the braid word for $b$ has length $m$. If the $j$-th $(1 \leq j \leq m)$ letter is $\sigma_{i}$ (resp. $\sigma_{i}^{-1}$ ) then we join the disks $\delta_{i}$ and $\delta_{i+1}$ by a positively (resp. negatively) twisted band embedded in the set of pages $\left\{A_{\theta} \mid(j-1) / m<\theta<j / m\right\}$. See Figure 8 (1).

(1)

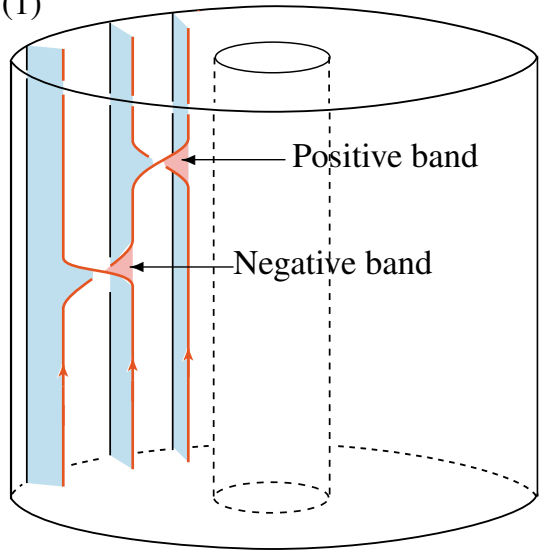

(2)

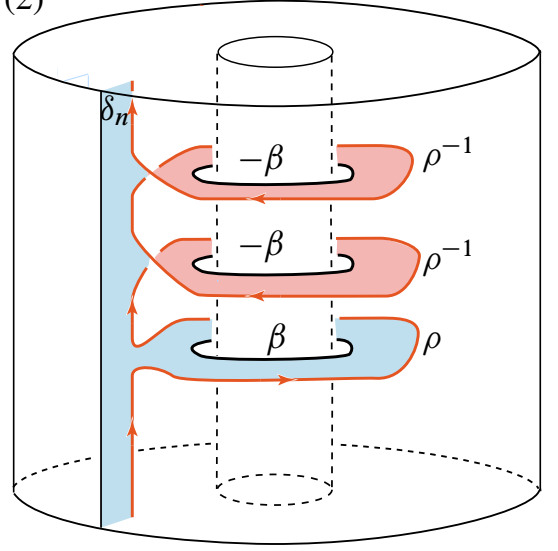

Figure 8: Construction of $F_{b}$ (1) Twisted bands (2) $\mathfrak{A}$-annuli 
If the $j$-th letter is $\rho$ (resp. $\rho^{-1}$ ), then we attach to the disk $\delta_{n}$ an annulus embedded in $\left\{A_{\theta} \mid(j-1) / m<\theta<j / m\right\}$. We call such an annulus an $\mathfrak{A}$-annulus. See Figure 8 (2). Let $\beta \subset A$ be an oriented circle between circles $\alpha$ and $\gamma$ as sketched in Figure 3. One of the boundaries of each $\mathfrak{A}$-annulus represents $\rho$ (resp. $\rho^{-1}$ ) and becomes part of the braid $b$. The other boundary, which we denote by $\beta_{j}$ (resp. $-\beta_{j}$ ), is in $\beta \times((j-1) / m, j / m)$.

We call the resulting surface $F_{b}$.

By [10, Proposition 4.6.11], we may assume that the characteristic foliation of our surface is of Morse-Smale type. Each disk $\delta_{i}$ has a positive elliptic point. A positive (negative) band between the $\delta$-disks contributes one positive (negative) hyperbolic point. The foliation on the disk $\delta_{n}$ together with an attached $\mathfrak{A}$-annulus has a positive (resp. negative) hyperbolic singularity as sketched in Figure 9 (1) (resp. (2)) if the corresponding braid word is $\rho$ (resp. $\left.\rho^{-1}\right)$.

(1)

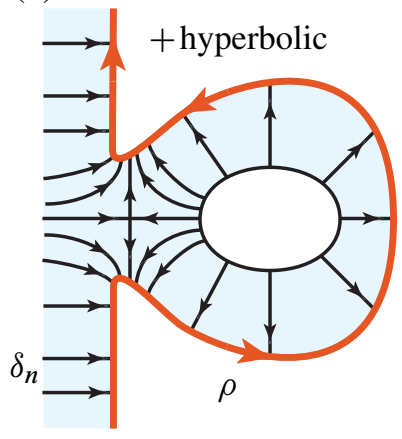

(2)

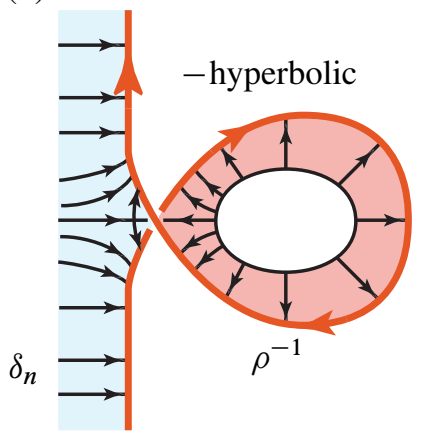

Figure 9: Characteristic foliations of $\mathfrak{A}$-annulus for (1) $\rho$ and (2) $\rho^{-1}$

\subsection{Construction of the surface $\tilde{F}_{b}$}

In Section 3.1, we have constructed an embedded oriented surface $F_{b}$ whose boundary consists of the braid $b$ and copies of $\pm \beta$ 's. Let $a_{\sigma} \in \mathbb{Z}$ (resp. $a_{\rho} \in \mathbb{Z}$ ) be the exponent sum of $\sigma_{1}, \ldots, \sigma_{n-1}$ (resp. $\rho_{1}, \ldots, \rho_{n-1}$ ) in the braid word for $b$. Let $r$ be the number of $\rho^{ \pm}$'s appearing in the braid word for $b$ of length $m$ (ie, $0 \leq r \leq m$ ). Then there exist $1 \leq j_{1}<\cdots<j_{r} \leq m$ and $\epsilon_{i}= \pm 1$ with $\epsilon_{1}+\epsilon_{2}+\cdots+\epsilon_{r}=a_{\rho}$ such that

$$
\partial F_{b}=b \cup \epsilon_{1} \beta_{j_{1}} \cup \epsilon_{2} \beta_{j_{2}} \cup \cdots \cup \epsilon_{r} \beta_{j_{r}} .
$$


Proposition 3.1 By attaching vertical annuli to pairs of $\beta$ and $-\beta$ circles as described in Figure 10, we can construct an embedded oriented surface, which we call $\widetilde{F}_{b}$, whose boundary consists of

$$
\partial \widetilde{F}_{b}= \begin{cases}b \text { and } a_{\rho} \text { copies of } \beta & \text { if } k>0, \\ b & \text { if } k=0, \\ b \text { and }-a_{\rho} \text { copies of }-\beta & \text { if } k<0 .\end{cases}
$$

(1)

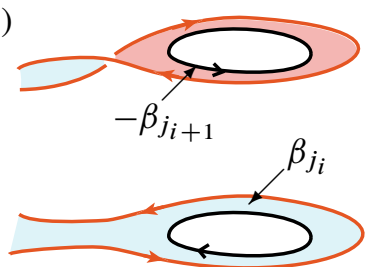

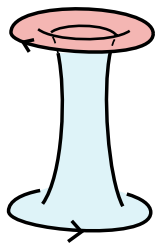

(2)

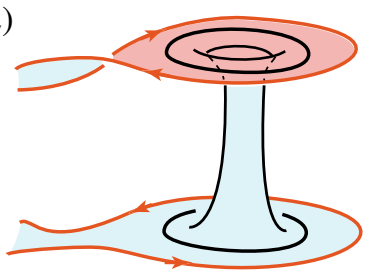

Figure 10: Attaching a vertical annulus to $\mathfrak{A}-$ annuli

Proof Suppose that $\partial F_{b}=b \cup \epsilon_{1} \beta_{j_{1}} \cup \epsilon_{2} \beta_{j_{2}} \cup \cdots \cup \epsilon_{r} \beta_{j_{r}}$ and $\epsilon_{1}+\epsilon_{2}+\cdots+\epsilon_{r}=a_{\rho}$. If $\epsilon_{1}=\epsilon_{2}=\cdots=\epsilon_{r}\left(\rho\right.$ and $\rho^{-1}$ do not coexist in the braid word for $\left.b\right)$, then take $\widetilde{F}_{b}=F_{b}$.

Else, let $1 \leq i \leq r-1$ be the smallest index for which $\epsilon_{i} \neq \epsilon_{i+1}$. We attach a "vertical" annulus to $\left(\epsilon_{i} \beta_{j_{i}}\right) \cup\left(\epsilon_{i+1} \beta_{j_{i+1}}\right)$ as sketched in Figure 10 . The boundary of the newly obtained surface (call this surface $F_{b, 1}$ ) contains two less $\pm \beta$-curves:

$$
\partial F_{b, 1}=b \cup \epsilon_{1} \beta_{j_{1}} \cup \cdots \cup \epsilon_{i-1} \beta_{j_{i-1}} \cup \epsilon_{i+2} \beta_{j_{i+2}} \cup \cdots \cup \epsilon_{r} \beta_{j_{r}},
$$

but it preserves the sum: $\epsilon_{1}+\cdots+\epsilon_{i-1}+\epsilon_{i+2}+\cdots+\epsilon_{r}=a_{\rho}$.

Renumber the boundary components,

$$
\partial F_{b, 1}=b \cup \epsilon_{1} \beta_{j_{1}} \cup \epsilon_{2} \beta_{j_{2}} \cup \cdots \cup \epsilon_{r-2} \beta_{j_{r-2}}
$$

then repeat the procedure for $F_{b, 1}$. If $i-1$ is the smallest index for which $\epsilon_{i-1} \neq \epsilon_{i}$, then attach the annulus by nesting it inside the one previously attached. See the right sketch in Figure 11. After at most $[r / 2]$ such attachments of annuli, all the $\epsilon_{i}$ 's have the same sign, and we get the desired surface $\widetilde{F}_{b}$. By Proposition 2.6 and Corollary 2.9 we have the equality (3-1). 

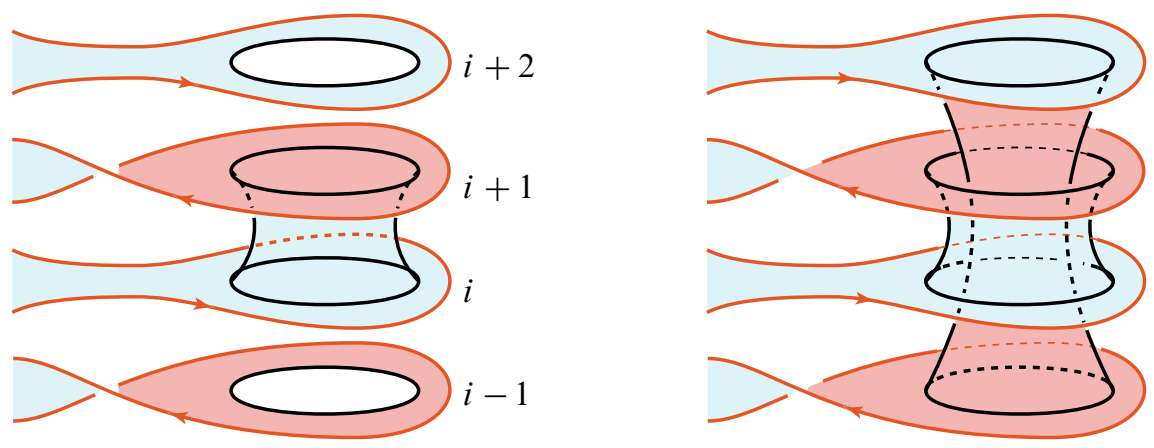

Figure 11: Nested vertical annuli

\subsection{Construction of the immersed surface $\check{\boldsymbol{F}}_{\boldsymbol{b}}$}

We have constructed a surface $\widetilde{F}_{b}$ satisfying the boundary condition (3-1). In particular, when $k=0$ we have already obtained an embedded surface $\widetilde{F}_{b}$ whose boundary is $b$. Define $\Sigma_{b}:=\widetilde{F}_{b}$.

When $k \neq 0$, we construct an immersed surface $\check{F}_{b}$ from $\widetilde{F}_{b}$, by attaching disks about the binding $\gamma^{\prime}$.

Assume that $k>0$. Proposition 2.6 justifies assuming $a_{\rho} \geq 0$. Let $\mathfrak{A}_{1}, \ldots, \mathfrak{A}_{a_{\rho}} \subset \widetilde{F}_{b}$ be the $\mathfrak{A}$-annuli whose boundaries contribute to the $a_{\rho}$ copies of $\beta$-circles as in Proposition 3.1. Recall the number $s=a_{\rho} / k \geq 0$ defined in Corollary 2.9. Let $u_{1}, \ldots, u_{s} \subset A$ be arcs (see Figure 3) disjoint from the Dehn twist circle $\alpha$, such that one end of each $u_{i}$ sits on the binding $\gamma^{\prime}$. Let $\omega_{1}, \ldots, \omega_{s}$ be disks, called $\omega-$ disks, obtained by swiping $u_{1}, \ldots, u_{s}$ in the open book $\left(A, D^{k}\right)$ so that the center of $\omega_{i}$ is pierced by $\gamma^{\prime}$. For each $i=1, \ldots, s$, connect $\omega_{i}$ smoothly with annuli $\mathfrak{A}_{i}, \mathfrak{A}_{s+i}, \mathfrak{A}_{2 s+i}, \ldots, \mathfrak{A}_{(k-1) s+i}$ by $k$ copies of the twisted band as in Figure 12 (1). When $k<0$, attach twisted bands as in Figure 12 (2). We have obtained an immersed surface, which we denote by $\check{F}_{b}$; see Figure 13 .

Lemma 3.2 Regardless of the sign of $k$, all the singularities for the characteristic foliation of the attached bands and the $\omega$-disks are positive elliptic.

Remark 3.3 The surface $\check{F}_{b}$ has $s$ additional positive elliptic points compared to $\widetilde{F}_{b}$.

Proof By definition of $\omega$-disk, its characteristic foliation has a single singularity at its center and it is of elliptic type (Figure 12). The orientation of $\omega$-disk is induced from that of the $\mathfrak{A}$-annuli so that the sign of the elliptic point is positive regardless of the sign of $k$. 

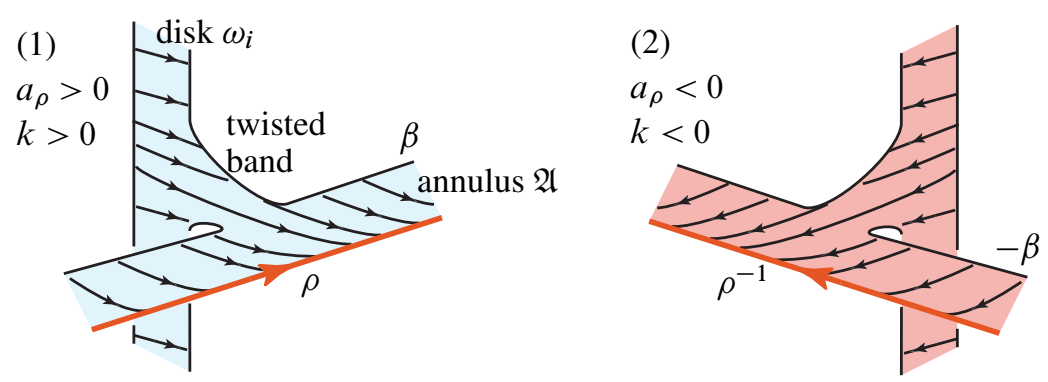

Figure 12: An $\omega$-disk and an $\mathfrak{A}$-annulus joined by a twisted brand

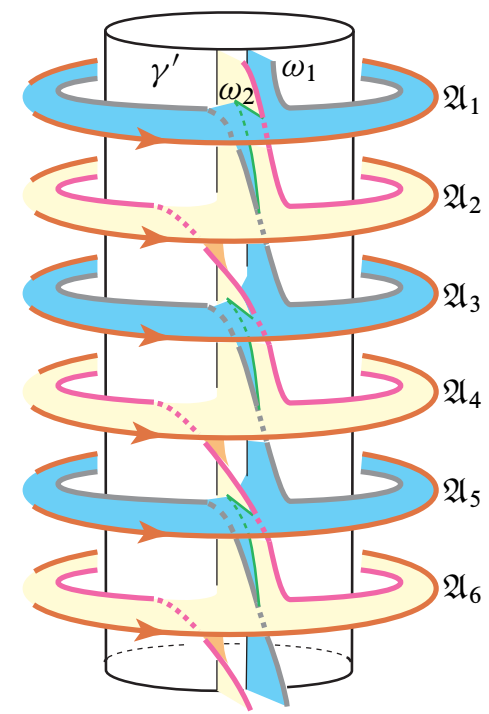

Figure 13: A part of immersed surface $\check{F}_{b}$ for $k=3, a_{\rho}=6, s=2$. Two $\omega$-disks and six $\mathfrak{A}$-annuli joined by twisted bands. Self-intersections are marked by thin green curves.

In the following, we show that there are no hyperbolic points on the twisted band. See Figure 14. Parameterize the twisted band as $[0,1] \times[-1,1]$. Attach the side $\{0\} \times[-1,1]$ of the band to the $\omega$-disk and $\{1\} \times[-1,1]$ to the $\beta$-circle so that the (dashed) line segment $[0,1] \times\{1 / 2\}$ sit on one page of the open book. We make the resulting surface smooth near the two attaching sides of the band. Take points on the twisted band $p_{1}=(0,1 / 2), p_{2}=(1 / 2,1 / 2)$, and $p_{3}=(1,1 / 2)$. Let $v_{i}$ be a tangent vector (red arrow) of the band at $p_{i}$ that is perpendicular to the dashed line $[0,1] \times\{1 / 2\}$.

When $k>0$, with respect to the page of the open book, $v_{1}$ is vertical, $v_{2}$ is slanted $45^{\circ}$, and $v_{3}$ is slanted $\epsilon$-degree $(0<\epsilon<45)$ because the braid $b$ transversely intersects all 

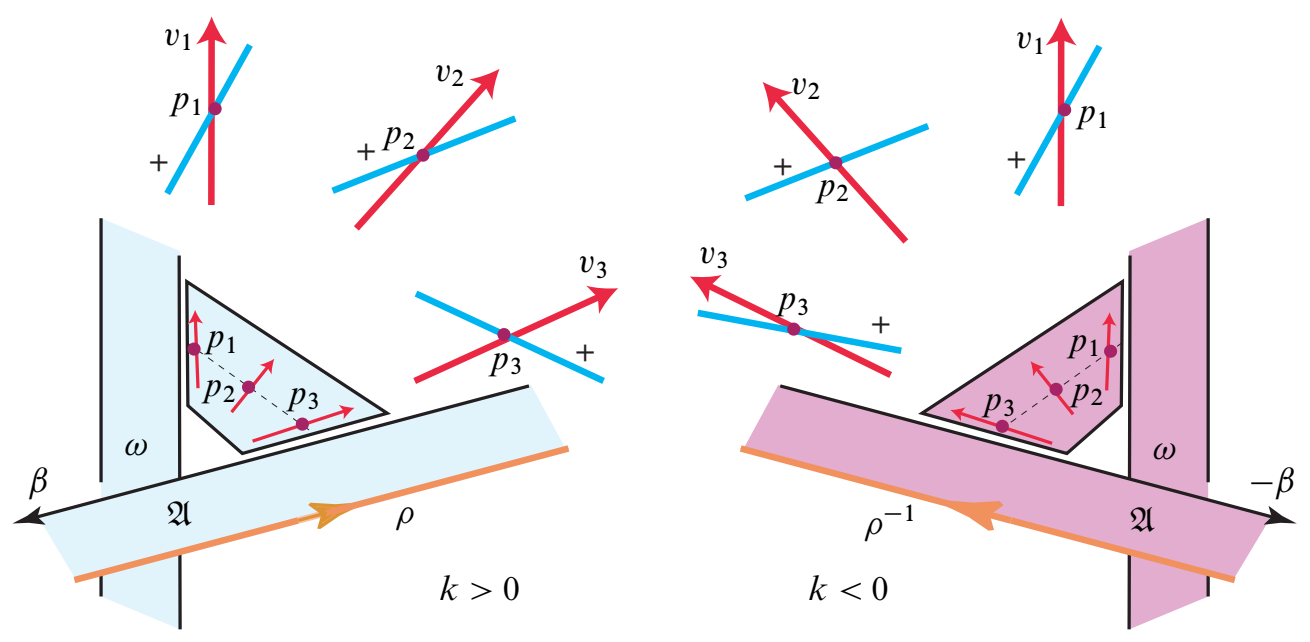

Figure 14: Proof of Lemma 3.2

the pages of the open book positively. Next we look at contact planes $\xi_{p_{i}}$ (light blue line segment) at $p_{i}$. In Figure 14, the positive side of a contact plane is marked "+". At each point of the bindings $\gamma, \gamma^{\prime}$, we may assume that the contact plane is positively perpendicular to the binding. Between the bindings, the contact planes rotate $180^{\circ}$ counter clockwise along the radial lines. Since $p_{1}$ is close to the binding $\gamma^{\prime}$, for some $\epsilon_{1}>0, \xi_{p_{1}}$ is slanted $\left(90-\epsilon_{1}\right)$-degree with respect to the page of the open book. While, $\xi_{p_{3}}$ is slanted $\left(-\epsilon_{3}\right)$-degree for some $\epsilon_{3}>0$ since $p_{3}$ is on the circle $\beta$ which is between $\alpha$ and $\gamma$ (Figure 3). At any point between $p_{1}$ and $p_{3}$ on the dashed line, the tangent plane is slanted more than the contact plane. It means that they never coincide. Since the band is a small neighborhood of the dashed line, contact planes are never tangent to the band, hence there are no singularities in the characteristic foliation on the twisted band.

When $k<0$, at $p_{3}$, the tangent vector $v_{3}$ is slanted $(180-\epsilon)$-degree and the contact plane $\xi_{p_{3}}$ is slanted $\left(-\epsilon_{3}\right)$-degree. Braid $b$ is a transverse link so it intersects contact planes positively. Considering that $p_{3}$ is close to the braid (orange arc), $v_{3}$ intersects $\xi_{p_{3}}$ positively, ie, $\epsilon>\epsilon_{3}$. Therefore, the tangent planes and the contact planes never coincide along the dashed line from $p_{1}$ to $p_{3}$, hence there are no singularities in the characteristic foliation on the twisted band.

\subsection{Construction of the immersed surface $\widehat{F}_{b}$}

Let $\tau$ be a closed braid in $\left(A, D^{k}\right)$ of braid index $=1$. The immersed surface $\check{F}_{b}$ constructed in Section 3.3 has boundary $[b]+s[\tau+k \beta]$ in $H_{1}\left(M_{\left(A, D^{k}\right)} ; \mathbb{Z}\right)$. Each 
closed curve representing $-[\tau+k \beta]$ bounds a disk about the binding $\gamma$. We call it a $\mathcal{D}$-disk; see Figure 15. There, the spirals in the bottom annulus page are identified, via

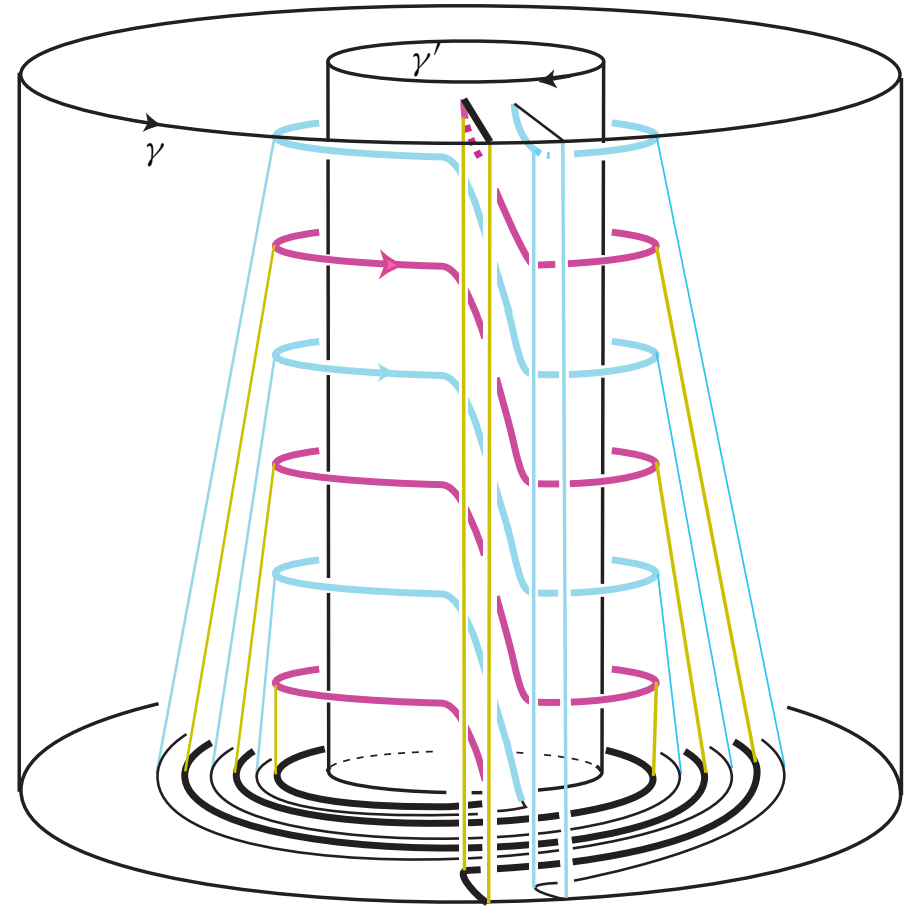

Figure 15: $\mathcal{D}$-disks

the Dehn twist $D^{k}$, with the straight line segments in the top annulus page. There are $s$ copies of $\mathcal{D}$-disk and they are disjoint from each other. Since $\mathcal{D}$-disks are nearly "vertical" as in Figure 15, the tangent planes and the contact planes, which rotate $180^{\circ}$ counter clockwise along the radial lines from $\gamma$ to $\gamma^{\prime}$, intersect transversely. This means that the characteristic foliation of each $\mathcal{D}$-disk has a single singularity, which occurs at the intersection point with $\gamma$ and whose type is elliptic. The orientations of the $\mathcal{D}$-disks are compatible with those of the boundaries so that the $\mathcal{D}$-disks and $\gamma$ intersect negatively. Therefore the signs of the elliptic points are negative.

Definition 3.4 We construct an immersed surface $\widehat{F}_{b}$ by gluing the $\mathcal{D}$-disks and $\check{F}_{b}$ along the $s$ copies of the $\tau+k \beta$ curve.

Remark 3.5 This $\widehat{F}_{b}$ has $s$ additional negative elliptic singularities given by the $\mathcal{D}$-disks compared to the surface $\check{F}_{b}$. 


\subsection{Resolution of singularities}

We start this section by defining three types of intersection of surfaces - branch, clasp and ribbon - then study resolution of self-intersections.

Definition 3.6 Let $\Sigma$ be an immersed oriented surface with $\partial \Sigma=K$ given by the immersion $i: \widetilde{\Sigma} \rightarrow \Sigma$. Let $l \subset \Sigma$ be a simple arc where $\Sigma$ intersects itself, and denote by $p$ and $q$ the endpoints of $l$.

- If $p$ is sitting on $K$, and $q$ is a branch point of a neighborhood Riemann surface; see Figure $16(1)$, then we call $l$ a branch intersection.
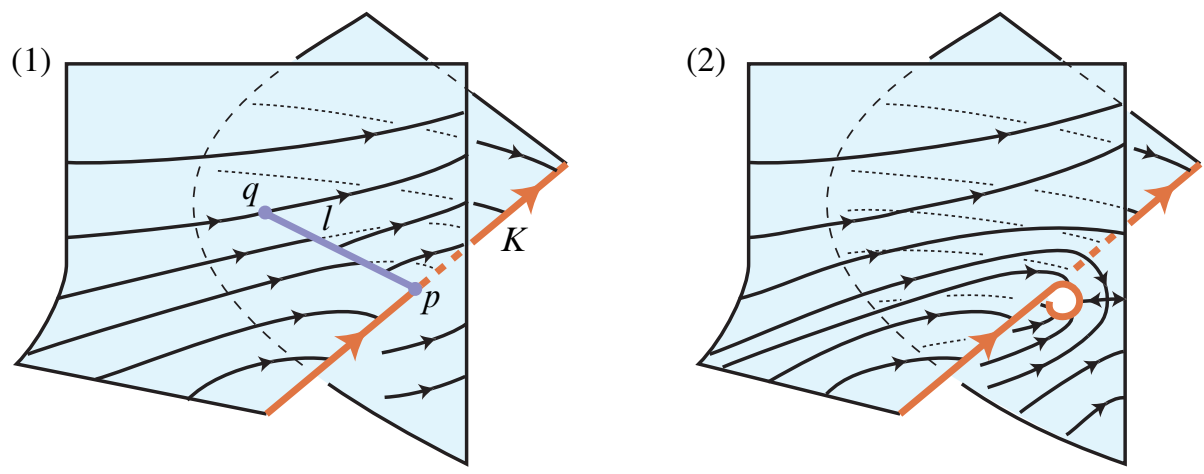

Figure 16: (1) A negative branch intersection $l$ and (2) its resolution

Next assume that the preimage of $l, i^{-1}(l) \subset \tilde{\Sigma}$, consists of two arcs, say $\tilde{l}_{1}, \tilde{l}_{2}$. Denote the end points of $\tilde{l}_{i}$ by $\tilde{p}_{i}$ and $\tilde{q}_{i}$ for $i=1,2$.

- If $\tilde{p}_{1}, \widetilde{q}_{2} \in \partial \Sigma$ and $\tilde{p}_{2}, \widetilde{q}_{1} \in \operatorname{Int}(\widetilde{\Sigma})$ then we call the intersection a clasp intersection. A local picture of $l$ is the left sketch of Figure 17.

- If $\tilde{p}_{1}, \widetilde{q}_{1} \in \partial \Sigma$ and $\tilde{p}_{2}, \widetilde{q}_{2} \in \operatorname{Int}(\tilde{\Sigma})$ then we call the intersection a ribbon intersection. See the right sketch of Figure 17.

Example 3.7 See Figure 18. The immersed surface, $\widehat{F}_{b}$, has:

- $\left|a_{\rho}\right|$ branches formed by $\mathfrak{A}$-annuli and $\mathcal{D}$-disks.

- $|k|\left(\begin{array}{l}s \\ 2\end{array}\right)=\frac{1}{2}\left|a_{\rho}\right|(s-1)$ clasp intersections when $s>1$. Recall that $\widehat{F}_{b}$ is obtained by attaching $s$ copies of $\mathcal{D}$-disk about the binding $\gamma$. Each pair among these $s$ disks interacts as in Figure 18 giving rise to $|k|$ clasp intersections. When $s=1,0$, there are no clasps.

- several ribbon intersections of $\mathcal{D}$-disks and the (nested) vertical annuli of Figure 10. 

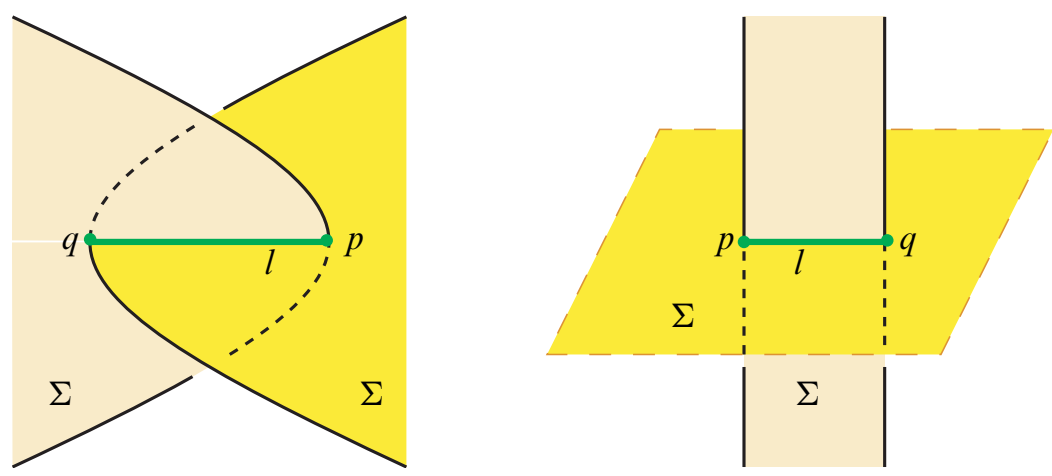

Figure 17: (Left) A clasp intersection (Right) A ribbon intersection

In Section 3.6, we resolve these self-intersections to obtain an embedded surface $\Sigma_{b}$. In the following, we assume that $K$ is a transverse knot in a contact manifold $(M, \xi)$ and $\Sigma$ an immersed oriented surface with $\partial \Sigma=K$. Also, we assume that (i) the self-intersection set of $\Sigma$ consists of ribbon, clasp, or branch intersections; (ii) the characteristic foliation $\mathcal{F}_{\Sigma}$ is of Morse-Smale type.

Let $l \subset \Sigma$ be a self-intersection arc. Near a point $x \in \operatorname{Int}(l), \Sigma$ intersects itself transversely as in Figure 19 (1). Let $F_{i} \subset \Sigma(i=1,2,3,4)$ be surfaces meeting at $l$. The orientation of $F_{i}$ is induced from that of $\Sigma$. Resolve the singularity $l$ by cutting $\Sigma$ out along $l$ and regluing $F_{1}, F_{2}$ along $l$ and $F_{3}, F_{4}$ along $l$ so that the orientations of the surfaces agree. See Figure 19 (2). Call the new surface $\Sigma^{\prime}$.

We orient the leaves of the characteristic foliation following Ozbagci and Stipsicz [14, page 80]: For $p \in \Sigma$ a nonsingular point of a leaf $L$ of the foliation, let $\vec{n} \in T_{p} \Sigma$ be a positive normal vector to $\xi_{p}$. We choose a vector $\vec{v} \in T_{p} L$ so that $(\vec{v}, \vec{n})$ is a positive basis for $T_{p} \Sigma$. This vector field $\vec{v}$ determines the orientation of the characteristic foliation.

We observe that if both $\mathcal{F}_{F_{1}}$ and $\mathcal{F}_{F_{2}}$ transversely intersect the line $l$ (Figure 19 (1)), then the orientations of $\mathcal{F}_{F_{1}}$ and $\mathcal{F}_{F_{2}}$ agree at $l$. Hence, after the cut and glue operation, the new characteristic foliation $\mathcal{F}_{\Sigma^{\prime}}$ is obtained by smoothly connecting the old $\mathcal{F}_{F_{1}}$ and $\mathcal{F}_{F_{2}}$, and also $\mathcal{F}_{F_{3}}$ and $\mathcal{F}_{F_{4}}$. See Figure 19 (2).

Near the endpoints of $l$, this resolution creates new hyperbolic points and $\mathcal{F}_{\Sigma^{\prime}}$ can be made into Morse-Smale type. See Figure 16 (2) and Figure 20 (2). The signs of the new hyperbolic points are determined in the following way:

Proposition 3.8 Suppose that $p \in \partial l \cap K$ and both $\mathcal{F}_{F_{1}}$ and $\mathcal{F}_{F_{2}}$ are transversely intersecting with $l$. If $p$ is a positive (negative) transverse intersection of $K$ and $\Sigma$, then the new hyperbolic point has positive (negative) sign. 

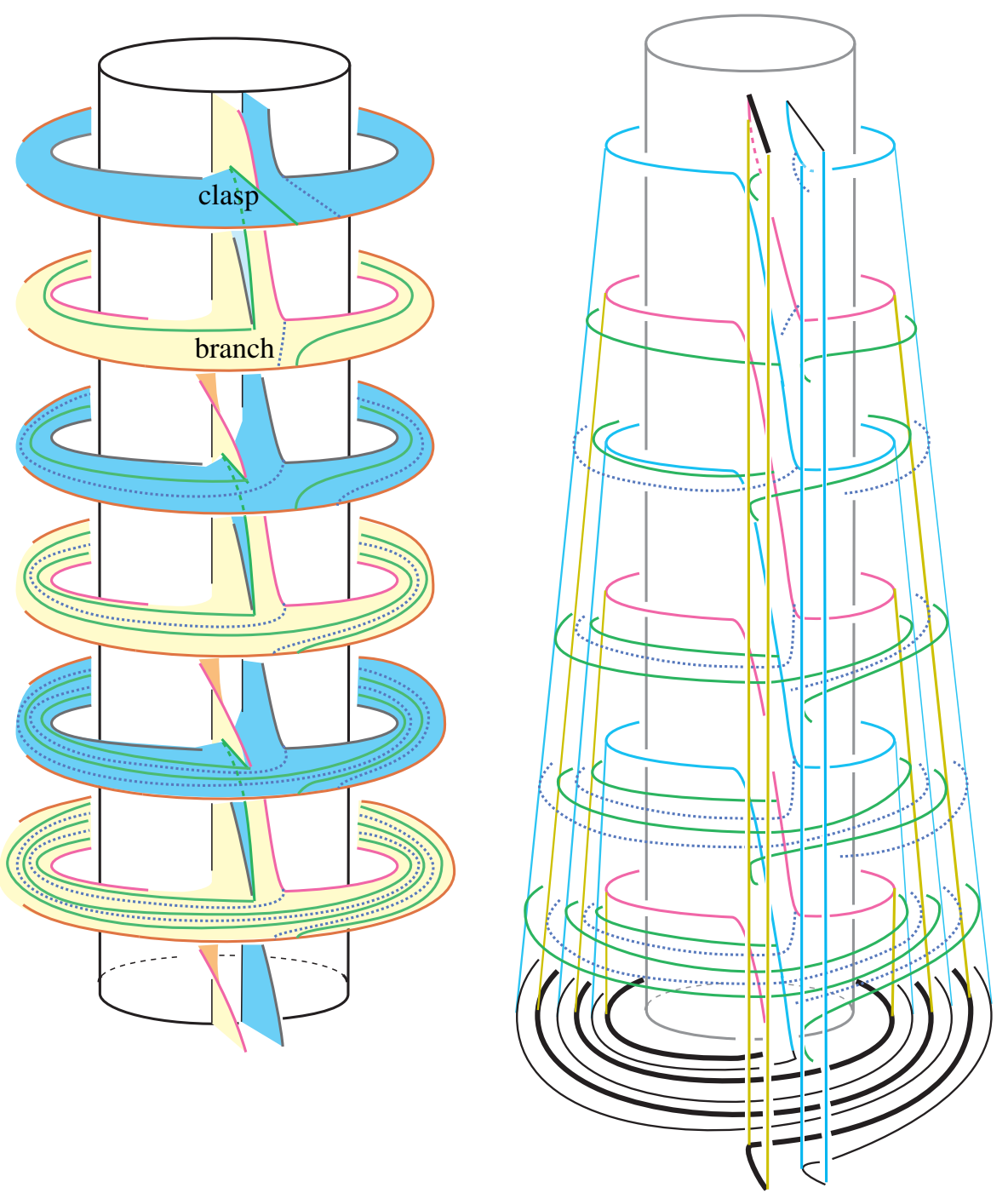

Figure 18: Clasp (green) and branch (dashed purple) intersections on $\widehat{F}_{b}$

Proof Assume that $p$ is a negative intersection, as depicted in Figure 20 (1). We introduce an $(x, y, z)$-coordinate system for a small neighborhood $N$ of $p$ : Identify $p$ with $(0,0,0)$, and identify $-K$ with the $z$-axis. Regard the surface which $K$ penetrates as the $x y$-plane. Since $K$ is a transverse knot, it transverses the contact 2-planes positively. Thus at a point $r \in K \cap N$ the positive normal vector $\vec{n}_{r}$ to the contact plane $\xi_{r}$ has a negative $z$-component, ie, $\vec{n}_{r} \cdot(0,0,1)<0$. We may assume that contact 


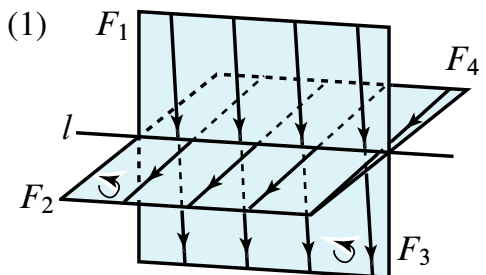

Figure 19: (1) Immersed surface $\Sigma$ singularity $l$

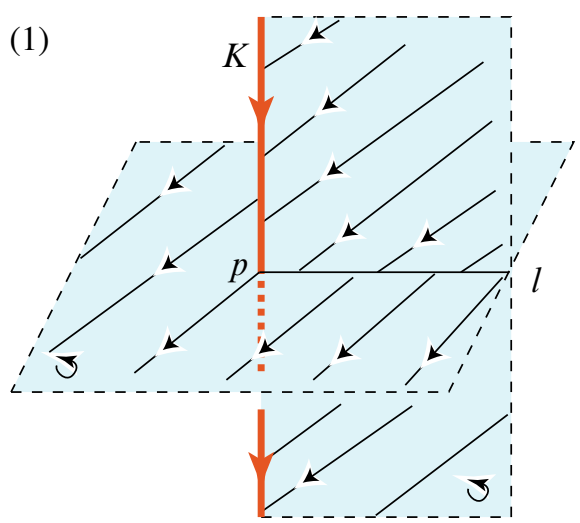

Figure 20: (1) A negative intersection $p$ (2) Creation of a negative hyperbolic singularity $h$ by resolving singular arc $l$
(2)

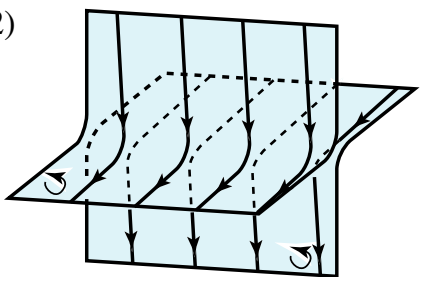

(2) New surface $\Sigma^{\prime}$ after resolution of

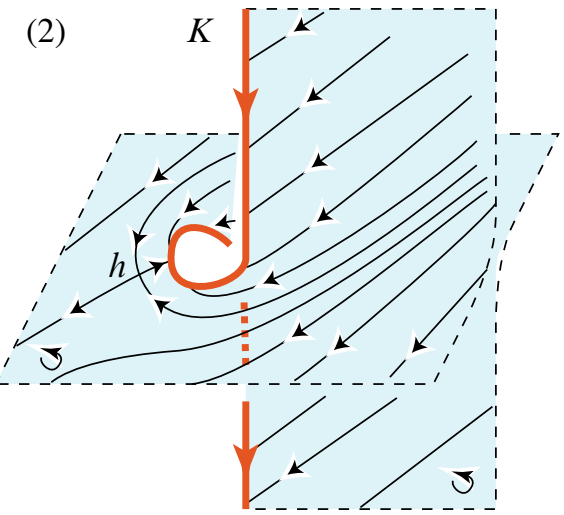

planes are almost parallel to each other in $N$. Therefore, at the new hyperbolic point $h \in N$ we have $T_{h} \Sigma=-\xi_{h}$. This means that $h$ is a negative hyperbolic point.

Since the two end points of a ribbon (resp. clasp) singularity have the same sign (resp. opposite signs), it follows that:

Corollary 3.9 (1) Resolution of a ribbon singularity creates one positive and one negative hyperbolic points.

(2) Resolution of a clasp singularity creates two hyperbolic points of the same sign.

(3) Resolution of a branch singularity creates one hyperbolic point. See Figure 16.

It makes sense to define the sign for clasp and branch arcs:

Definition 3.10 (1) If both end points of a clasp arc are positive (negative) intersections of $K$ and $\Sigma$, then we say the sign of the clasp is positive (negative).

(2) If the end point $p=l \cap K$ of a branch arc is a positive (negative) intersection, then we say the sign of the branch arc is positive (negative). 


\subsection{Construction of the embedded surface $\Sigma_{b}$}

In Section 3.3 we have defined a Seifert surface $\Sigma_{b}$ for the case $k=0$.

When $k \neq 0$, Example 3.7 shows that the immersed surface $\widehat{F}_{b}$ has branch, clasp, and ribbon intersections. In this section, we construct an embedded surface $\Sigma_{b}$ by resolving these singularities.

When $k>0$, as shown in Figure 21 (1)-(2), we can make all the branch, ribbon and clasp arcs transverse to the characteristic foliation $\mathcal{F}_{\widehat{F}_{b}}$. We apply the argument in Section 3.5 and construct an embedded surface $\Sigma_{b}$. Since all the signs of the branch and clasp arcs are negative, Example 3.7 and Corollary 3.9 imply that, when $s>0$, the resolution of these self-intersections creates, in total, $a_{\rho}+2\left(\frac{1}{2} a_{\rho}(s-1)\right)=a_{\rho} s$ negative hyperbolic singularities. When $s=0$ there are no branch or clasp intersections, so no new hyperbolic points are created.

When $k<0$, a similar argument holds.
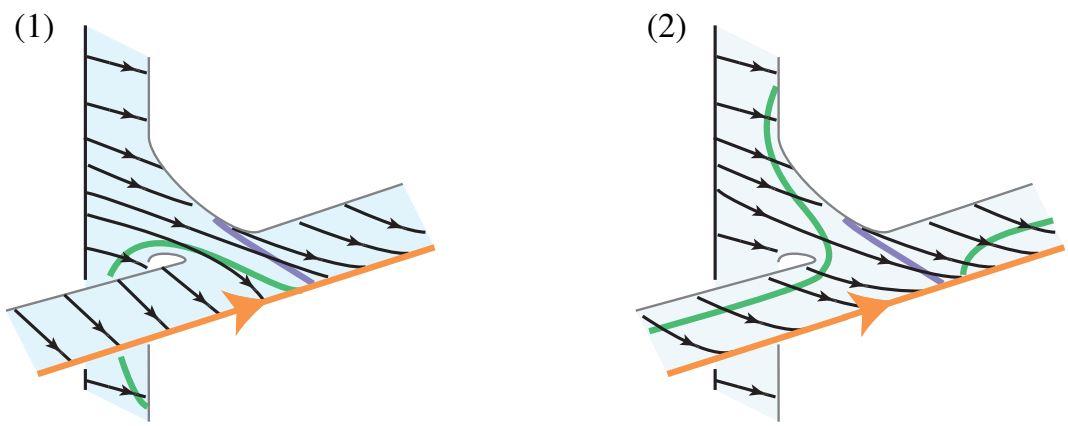

(3)

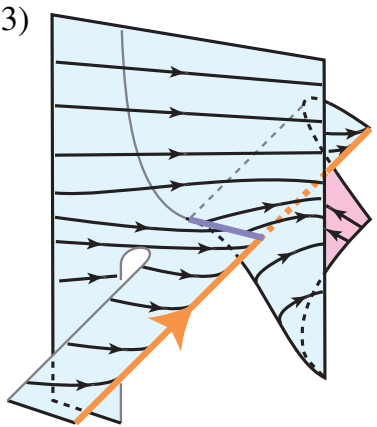

(4)

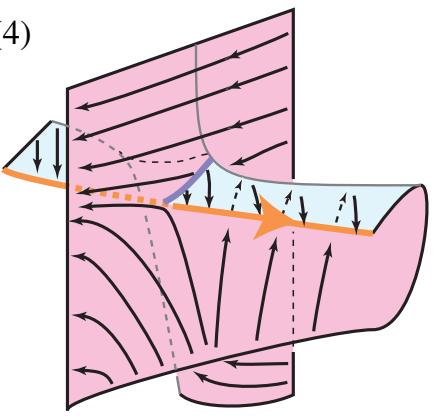

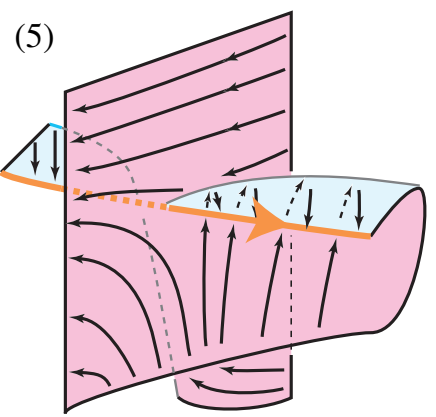

Figure 21: Clasp (light green) and branch (dark purple) intersections are transverse to characteristic foliations (top). Characteristic foliation near a branch singularity and its resolution (bottom). 


\section{The self-linking number}

We finally compute the self-linking number $\operatorname{sl}\left(b,\left[\Sigma_{b}\right]\right)$ of $b$ relative to the embedded surface $\Sigma_{b}$.

Theorem 4.1 Let $b$ be a null-homologous transverse braid in the open book decomposition $\left(A, D^{k}\right)$ satisfying Assumption 2.3. There exists a Seifert surface $\Sigma_{b}$ of $b$ which satisfies the formula

$$
\operatorname{sl}\left(b,\left[\Sigma_{b}\right]\right)=-n+a_{\sigma}+a_{\rho}(1-s) .
$$

In particular, when $k \neq 0, \operatorname{sl}\left(b,\left[\Sigma_{b}\right]\right)$ does not depend on the choice of Seifert surface and hence we have the general formula

$$
\operatorname{sl}(b)=-n+a_{\sigma}+a_{\rho}(1-s) .
$$

Remark 4.2 - When $k \neq 0$, our manifold $M_{\left(A, D^{k}\right)}$ is a lens space (Claim 2.1) which has $H_{2}(L(k, q) ; \mathbb{Z})=0$, ie, the self-linking number does not depend on choice of a Seifert surface and we can denote $\operatorname{sl}\left(b,\left[\Sigma_{b}\right]\right)$ simply by $\operatorname{sl}(b)$.

- The null-homologous condition ensures $a_{\rho}=0$ when $k=0$ (Corollary 2.9).

- When $a_{\rho}=0$ we exactly obtain Bennequin's formula (1-1).

Proof of Theorem 4.1 Let $\Sigma_{b}$ be the surface constructed in Section 3.6.

It is known (see Etnyre [6] for example) that

$$
\operatorname{sl}\left(b,\left[\Sigma_{b}\right]\right)=-\left(e^{+}-e^{-}\right)+\left(h^{+}-h^{-}\right),
$$

where $e^{+}\left(e^{-}\right)$and $h^{+}\left(h^{-}\right)$represent the number of positive (negative) elliptic and positive (negative) hyperbolic singularities of the characteristic foliation $\mathcal{F}_{\Sigma_{b}}$ on $\Sigma_{b}$. Let $h_{\sigma}^{+}\left(h_{\sigma}^{-}\right)$be the number of $\sigma_{i}$ 's ( $\sigma_{i}^{-1}$ 's) which appear in the braid word for $b$. Then we have $a_{\sigma}=h_{\sigma}^{+}-h_{\sigma}^{-}$, the sign count of hyperbolic singularities on $\Sigma_{b}$ given by the bands joining $\delta$-disks as in Figure 8 (1).

Based on Remarks 3.3, 3.5 and Section 3.6, we summarize the count of singularities:

$e^{+}=(n ;$ on $\delta$-disks $)+(s ;$ on $\omega$-disks $)$

$e^{-}=(s ;$ on $\mathcal{D}$-disks $)$

$h^{+}=\left(h_{\sigma}^{+} ;\right.$on + bands between $\delta$-disks $)+\left(a_{\rho} ;\right.$ on bands between $\delta_{n}$ and $\mathfrak{A}$-annuli $)$

$h^{-}=\left(h_{\sigma}^{-}\right.$; on - bands between $\delta$-disks $)$ $+\left(a_{\rho} s\right.$; by resolution of branches, clasps, ribbons $)$

By (4-1) we obtain the desired formula. 
The Bennequin-Eliashberg inequality $[2 ; 4]$ states that the contact structure $(M, \xi)$ is tight if and only if

$$
\operatorname{sl}(K,[\Sigma]) \leq-\chi(\Sigma)
$$

for any $(K, \Sigma)$ a null-homologous transverse knot and its Seifert surface.

Corollary 4.3 The contact structure $\left(M_{\left(A, D^{k}\right)}, \xi_{k}\right)$ is tight if and only if for any braid $b \subset\left(A, D^{k}\right)$ inequality $\mathrm{sl}(b) \leq-\chi\left(\Sigma_{b}\right)$ holds.

Proof of Corollary 4.3 As $\chi\left(\Sigma_{b}\right)=\left(e^{+}+e^{-}\right)-\left(h^{+}+h^{-}\right)$, the inequality $\operatorname{sl}(b) \leq$ $-\chi\left(\Sigma_{b}\right)$ is equivalent to $0 \leq h^{-}-e^{-}=h_{\sigma}^{-}+s\left(a_{\rho}-1\right)$. Claim 2.2 states that $\left(M_{\left(A, D^{k}\right)}, \xi_{k}\right)$ is tight if and only if $k \geq 0$.

When $k \geq 0$, we have $s\left(a_{\rho}-1\right)=s(k s-1) \geq 0$, thus $h^{-}-e^{-} \geq 0$.

When $k<0$, we have $s\left(a_{\rho}-1\right)<0$ by Corollary 2.9. Therefore, there exists $b$ for which $h^{-}-e^{-}<0$.

Remark 4.4 It is interesting to note that the Bennequin-Eliashberg inequality is not satisfied for the immersed surface $\widehat{F}_{b}$ even for the tight cases.

Next, we study behavior of $\operatorname{sl}\left(b,\left[\Sigma_{b}\right]\right)$ under braid stabilizations.

Let $b$ be a null-homologous braid in the open book $\left(A, D^{k}\right)$. For $\epsilon \in\{+,-\}$ let $b_{\epsilon}^{\gamma}$ (resp. $b_{\epsilon}^{\gamma^{\prime}}$ ) denote the braid obtained from $b$ after an $\epsilon$-stabilization about the binding $\gamma$ (resp. $\gamma^{\prime}$ ). By Theorem 2.8, braids $b, b_{+}^{\gamma}$ and $b_{+}^{\gamma^{\prime}}$ are transversely isotopic regardless of choice of stabilization arc $a$. Etnyre's [5, Theorem 3.8] implies that if $b$ is a one component link, then a negative stabilization is unique up to transverse isotopy, regardless of choice of $\operatorname{arc} a$, thus $b_{-}^{\gamma}=b_{\underline{\gamma^{\prime}}}$.

Corollary 4.5 We have

$$
\begin{aligned}
& \operatorname{sl}\left(b,\left[\Sigma_{b}\right]\right)=\operatorname{sl}\left(b_{+}^{\gamma},\left[\Sigma_{b_{+}^{\nu}}\right]\right)=\operatorname{sl}\left(b_{-}^{\gamma},\left[\Sigma_{b \underline{\gamma}}\right]\right)+2, \\
& \operatorname{sl}\left(b,\left[\Sigma_{b}\right]\right)=\operatorname{sl}\left(b_{+}^{\gamma^{\prime}},\left[\Sigma_{b_{+}^{\nu^{\prime}}}\right]\right)=\operatorname{sl}\left(b_{-}^{\gamma^{\prime}},\left[\Sigma_{b \underline{\underline{\gamma}^{\prime}}}\right]\right)+2 .
\end{aligned}
$$

Proof of Corollary 4.5 A positive (negative) stabilization about $\gamma$ changes $n \rightarrow n+1$ and $a_{\sigma} \rightarrow a_{\sigma}+1\left(a_{\sigma} \rightarrow a_{\sigma}-1\right)$. Applying Theorem 4.1, we get (4-3).

For (4-4), as we have seen in the proof of Proposition 2.6, by a positive (negative) braid stabilization, we have the following data change:

$$
\begin{aligned}
& n \rightarrow n+1, \quad a_{\sigma} \rightarrow a_{\sigma}+1+2 a_{\rho}, \quad s \rightarrow s+1, \quad a_{\rho} \rightarrow a_{\rho}+k, \\
& \left(n \rightarrow n+1, \quad a_{\sigma} \rightarrow a_{\sigma}-1+2 a_{\rho}, \quad s \rightarrow s+1, \quad a_{\rho} \rightarrow a_{\rho}+k\right) .
\end{aligned}
$$


Applying Theorem 4.1, we have

$$
\begin{aligned}
\operatorname{sl}\left(b_{+}^{\gamma^{\prime}},\left[\Sigma_{b_{+}^{\gamma^{\prime}}}\right]\right) & =-(n+1)+\left(a_{\sigma}+1+2 a_{\rho}\right)+\left(a_{\rho}+k\right)(1-(s+1)) \\
& =-n+a_{\sigma}+a_{\rho}(1-s) \\
& =\operatorname{sl}\left(b,\left[\Sigma_{b}\right]\right) .
\end{aligned}
$$

A similar computation leads to $\operatorname{sl}\left(b_{-}^{\gamma^{\prime}},\left[\Sigma_{b \underline{\gamma^{\prime}}}\right]\right)+2=\operatorname{sl}\left(b,\left[\Sigma_{b}\right]\right)$.

\section{Seifert fibered manifolds}

Let $S$ be an oriented pair of pants with boundary circles $\gamma_{i}$ for $i=1,2,3$. See Figure 22. Let $\alpha_{i}$ be circles parallel to $\gamma_{i}$. Denote the positive Dehn twist about $\alpha_{i}$
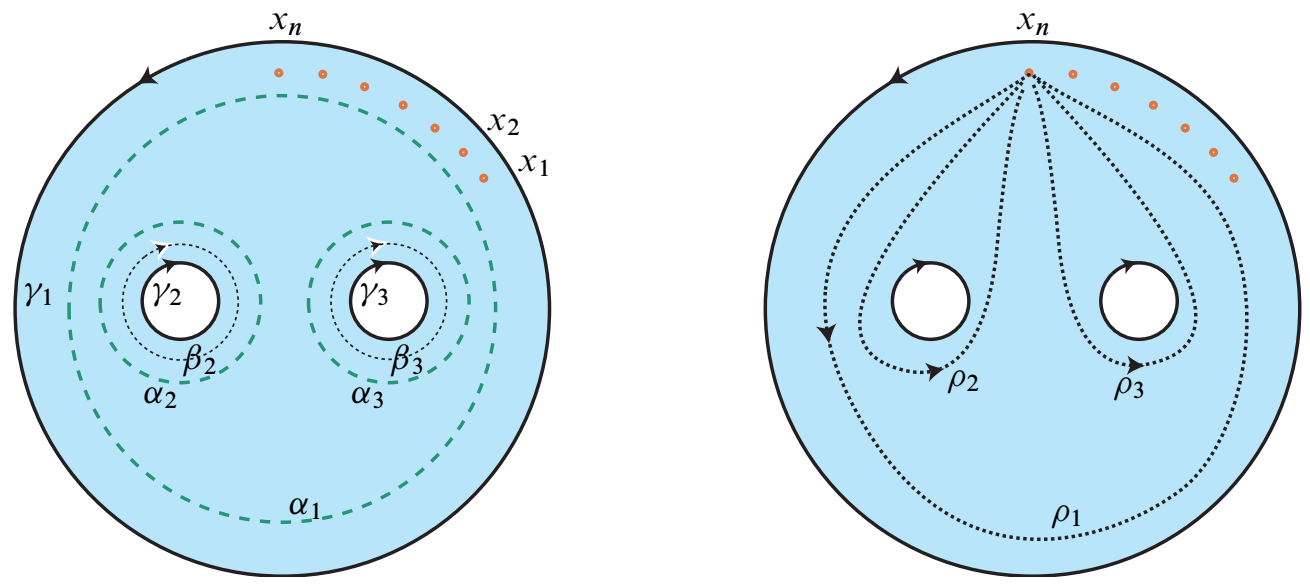

Figure 22: A pair of pants $S$

by $D_{i}$. Let $k_{i}$ be an integer, $i=1,2,3$. In this section we study closed braids in the open book decomposition $\left(S, D_{1}^{k_{1}} \circ D_{2}^{k_{2}} \circ D_{3}^{k_{3}}\right)$. The corresponding manifold, which we denote by $M_{k_{1}, k_{2}, k_{3}}(=M)$, is a Seifert fibered space over the orbifold of signature $\left(0, k_{1}, k_{2}, k_{3}\right)$. A similar argument as in the proof of Claim 2.1 tells that $M_{k_{1}, k_{2}, k_{3}}$ has surgery descriptions as in Figure 23. In Sketch (1), the two circles with slope 0 represent the unlinked unknots through the holes of $S, \gamma_{2}$ and $\gamma_{3}$ (cf the unknot $U$ in the proof of Claim 2.1). Slam-dunk operations are applied in the passage Sketch $(1) \rightarrow(2) \rightarrow(3)$. Etnyre and Ozbagci [8, page 3136] implies

$$
H_{1}\left(M_{k_{1}, k_{2}, k_{3}} ; \mathbb{Z}\right)=\left\langle c_{2}, c_{3} \mid\left(k_{1}+k_{2}\right) c_{2}+k_{1} c_{3}=k_{1} c_{2}+\left(k_{1}+k_{3}\right) c_{3}=0\right\rangle
$$




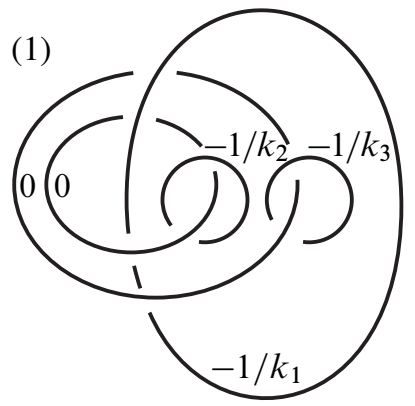

(2)

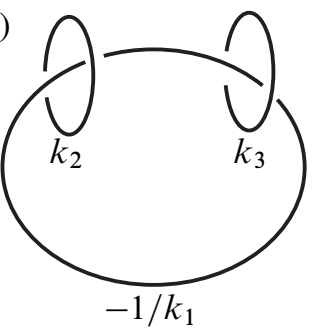

(3)

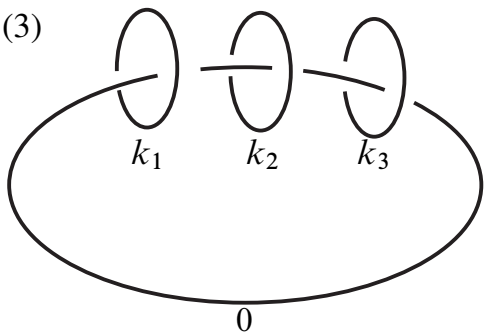

Figure 23: Surgery descriptions for $M_{k_{1}, k_{2}, k_{3}}$

where $c_{j} \in \pi_{1}(S)$ is the standard generator corresponding to the boundaries $\gamma_{j}$ of $S$. We remark that $H_{2}(M ; \mathbb{Z})$ is nontrivial in general, which is distinct from the lens spaces.

Proposition 5.1 Let $\xi_{k_{1}, k_{2}, k_{3}}$ denote the contact structure for $M_{k_{1}, k_{2}, k_{3}}$ compatible with the open book $\left(S, D_{1}^{k_{1}} \circ D_{2}^{k_{2}} \circ D_{3}^{k_{3}}\right)$ via the Giroux correspondence [11]. Then $\xi_{k_{1}, k_{2}, k_{3}}$ is tight if and only if $k_{1}, k_{2}, k_{3} \geq 0$.

Proof If $k_{1}=k_{2}=k_{3}=0$, then $M_{0,0,0}=\left(S^{1} \times S^{2}\right) \#\left(S^{1} \times S^{2}\right)$. Etnyre and Honda explain in the proof of [7, Lemma 3.2] that $\xi_{0,0,0}$ is Stein fillable, hence tight. In fact, it is the unique tight structure, which is due to Eliashberg [4].

If $k_{1}, k_{2}, k_{3} \geq 0$ and $\left(k_{1}, k_{2}, k_{3}\right) \neq(0,0,0)$, Etnyre and Honda [7, Lemma 3.2] tells that $\xi_{k_{1}, k_{2}, k_{3}}$ is tight.

If one of $k_{i}$ is negative, say $k_{1}<0$, then a properly embedded boundary nonparallel essential arc whose both ends sit on $\gamma_{1}$ is a sobering arc; see [12, Definition 3.2]. Thus Goodman's [12, Theorem 1.2] implies that $\xi_{k_{1}, k_{2}, k_{3}}$ is overtwisted.

Let $K$ be a null-homologous transverse knot in $\left(M, \xi_{k_{1}, k_{2}, k_{3}}\right)$. By Theorem 1.3 we can identify $K$ with a closed $n$-braid $b$ in $\left(S, D_{1}^{k_{1}} \circ D_{2}^{k_{2}} \circ D_{3}^{k_{3}}\right)$.

Assumption 5.2 Applying braid isotopy (transverse isotopy), we may assume that there exist points $x_{1}, \ldots, x_{n}$ (orange dots in Figure 22) sitting between $\gamma_{1}$ and $\alpha_{1}$ such that $b \cap(S \times\{0\})=\left\{x_{1}, \ldots, x_{n}\right\}$.

Let $\sigma_{i}$ be a mapping class of $S$ with $n$ fixed points $x_{1}, \ldots, x_{n}$, exchanging $x_{i}$ and $x_{i+1}$ counter clockwise. Let $\rho_{j}(j=1,2,3)$ be a mapping class which moves $x_{n}$ around the boundary circle $\gamma_{j}$ as described in Figure 22. Since $\sigma_{i}$ 's and $\rho_{j}$ 's are generators of the mapping class group and $\rho_{1}=\rho_{2}+\rho_{3}$, it follows that: 
Proposition 5.3 An $n$-strand closed braid in the open book $\left(S, D_{1}^{k_{1}} \circ D_{2}^{k_{2}} \circ D_{3}^{k_{3}}\right)$ can be written in letters $\left\{\sigma_{1}, \ldots, \sigma_{n-1}, \rho_{2}, \rho_{3}\right\}$.

We define symbols $a_{\sigma}, a_{\rho_{i}}$ and $s_{i}$ :

Definition 5.4 Let $a_{\sigma}$ be the exponent sum of $\sigma_{i}$ 's in the braid word for $b$. Let $a_{\rho_{i}}(i=2,3)$ be the exponent sum of $\rho_{i}$ in the braid word for $b$. Since $b$ is nullhomologous, we have $0=[b]=a_{\rho_{2}} c_{2}+a_{\rho_{3}} c_{3}$ in $H_{1}(M ; \mathbb{Z})$. By equation (5-1), there exist $s_{2}, s_{3} \in \mathbb{Z}$, so that

$$
0=[b]=s_{2}\left\{\left(k_{1}+k_{2}\right) c_{2}+k_{1} c_{3}\right\}+s_{3}\left\{k_{1} c_{2}+\left(k_{1}+k_{3}\right) c_{3}\right\} .
$$

Therefore,

$$
\left[a_{\rho_{2}}, a_{\rho_{3}}\right]=\left[s_{2}, s_{3}\right]\left[\begin{array}{cc}
k_{1}+k_{2} & k_{1} \\
k_{1} & k_{1}+k_{3}
\end{array}\right]
$$

For special cases,

(1) when $k_{1}=k_{2}=0$ and $k_{3} \neq 0$, we set $s_{2}=0$, ie, $a_{\rho_{2}}=0$ and $a_{\rho_{3}}=s_{3} k_{3}$,

(2) when $k_{1}=k_{3}=0$ and $k_{2} \neq 0$, we set $s_{3}=0$, ie, $a_{\rho_{3}}=0$ and $a_{\rho_{2}}=s_{2} k_{2}$,

(3) when $k_{1}=k_{2}=k_{3}=0$, we set $s_{2}=s_{3}=0$, ie, $a_{\rho_{2}}=a_{\rho_{3}}=0$.

Lemma 5.5 We may assume that $s_{2}, s_{3} \geq 0$ and that $a_{\rho_{2}}, a_{\rho_{3}}$ satisfy (5-2).

Proof A similar argument as in the proof of Proposition 2.6 applies. Recall that a positive braid stabilization preserves the transverse knot type (Theorem 2.8). Since the point $x_{n}$ is between $\gamma_{1}$ and $\alpha_{1}$, positive stabilizations of $b$ about $\gamma_{2}$ (resp. $\gamma_{3}$ ) for $\alpha$ times (resp. $\beta$ times), where $\alpha, \beta \geq 0$, change $a_{\rho_{i}}$ in the following way:

$$
\begin{aligned}
a_{\rho_{2}} & \mapsto a_{\rho_{2}}+\alpha\left(k_{1}+k_{2}\right), & a_{\rho_{3}} \mapsto a_{\rho_{3}}+\alpha k_{1}, \\
\text { (resp. } a_{\rho_{2}} \mapsto a_{\rho_{2}}+\beta k_{1}, & & \left.a_{\rho_{3}} \mapsto a_{\rho_{3}}+\beta\left(k_{1}+k_{3}\right)\right) .
\end{aligned}
$$

By (5-2), $s_{i}$ also changes as

$$
\begin{aligned}
s_{2} \mapsto s_{2}+\alpha & \text { if } k_{1}+k_{2} \neq 0 \text { or } k_{1} \neq 0, \\
\text { (resp. } s_{3} \mapsto s_{3}+\beta & \text { if } \left.k_{1}+k_{3} \neq 0 \text { or } k_{1} \neq 0\right) .
\end{aligned}
$$

Therefore taking $\alpha, \beta$ sufficiently large, we can make $s_{2}, s_{3} \geq 0$, even for the special three cases in Definition 5.4.

Now we state our main result of this section: 
Theorem 5.6 Let $b$ be a null-homologous closed braid in $\left(S, D_{1}^{k_{1}} \circ D_{2}^{k_{2}} \circ D_{3}^{k_{3}}\right)$ satisfying Assumption 5.2 and Lemma 5.5. There is a Seifert surface $\Sigma_{b}$ of $b$ for which the following holds:

$$
\operatorname{sl}\left(b,\left[\Sigma_{b}\right]\right)=-n+a_{\sigma}+a_{\rho_{2}}\left(1-s_{2}\right)+a_{\rho_{3}}\left(1-s_{3}\right)-\left(s_{2}+s_{3}\right) k_{1} .
$$

Proof We construct an embedded surface $\widetilde{F}_{b}$ after Sections 3.1 and 3.2.

- Construct $n$ copies of the $\delta$-disk (cf Figure 7).

- Join them by twisted bands for each $\sigma_{j}^{ \pm}$in the braid word (cf Figure 8 (1)).

- Attach an $\mathfrak{A}$-annulus for each $\rho_{2}^{ \pm}, \rho_{3}^{ \pm}$in the braid word (cf Figure 8 (2)).

- Attach vertical nested annuli to remove redundant boundaries (cf Figure 10). This procedure is more subtle than that of annulus open book case.

If $k_{1}, k_{2}, k_{3} \geq 0$ or $k_{1}, k_{2}, k_{3} \leq 0$ then attach vertical nested annuli near the bindings $\gamma_{2}$ and $\gamma_{3}$ following the algorithm described in the proof of Proposition 3.1. The boundary of the resulting surface, $\widetilde{F}_{b}$, has

$\partial \widetilde{F}_{b}=b \cup\left\{\left|\left(s_{2}+s_{3}\right) k_{1}+s_{2} k_{2}\right|\right.$ copies of $\left.\epsilon\left(k_{2}\right) \beta_{2}\right\}$

$$
\cup\left\{\left|\left(s_{2}+s_{3}\right) k_{1}+s_{3} k_{3}\right| \text { copies of } \epsilon\left(k_{3}\right) \beta_{3}\right\}
$$

where $\beta_{i}$ is the oriented circle as in Figure 22 and $\epsilon\left(k_{i}\right)$ is the sign of $k_{i}$.

Otherwise, by symmetry of the pants surface we may assume that (i) $k_{1}, k_{2} \geq 0$ and $k_{3}<0$, or (ii) $k_{1}, k_{2} \leq 0$ and $k_{3}>0$. For either case, we change the braid word by adding dummy letters that preserve the transverse knot type:

$$
b \mapsto\left(b \rho_{3}^{-s_{3} k_{3}}\right)\left(\rho_{3}^{s_{3} k_{3}}\right)
$$

Attach vertical nested annuli to the first part $b \rho_{3}^{-s_{3} k_{3}}$, without touching the remaining part $\rho_{3}^{s_{3} k_{3}}$, until all their boundary circles of the $\mathfrak{A}$-annuli have the same direction. The boundary of the resulting surface, $\widetilde{F}_{b}$, has

$$
\begin{aligned}
\partial \widetilde{F}_{b}=b \cup\left\{\left(s_{2}+s_{3}\right)\left|k_{1}\right|\right. & \text { copies of } \left.\epsilon\left(k_{2}\right)\left(\beta_{2}+\beta_{3}\right)\right\} \\
& \cup\left\{s_{2}\left|k_{2}\right| \text { copies of } \epsilon\left(k_{2}\right) \beta_{2}\right\} \cup\left\{s_{3}\left|k_{3}\right| \text { copies of } \epsilon\left(k_{3}\right) \beta_{3}\right\} .
\end{aligned}
$$

We require the operation (5-3) so that $\mathcal{D}$-disks introduced below can be compatible with the monodromy of the open book. Note that if the signs of $k_{2}$ and $k_{3}$ are different

$$
\text { but } \quad \begin{aligned}
\left|a_{\rho_{2}}\right| & =\left|\left(s_{2}+s_{3}\right) k_{1}+s_{2} k_{2}\right|=\left(s_{2}+s_{3}\right)\left|k_{1}\right|+s_{2}\left|k_{2}\right|, \\
\left|a_{\rho_{3}}\right| & =\left|\left(s_{2}+s_{3}\right) k_{1}+s_{3} k_{3}\right| \neq\left(s_{2}+s_{3}\right)\left|k_{1}\right|+s_{3}\left|k_{3}\right| .
\end{aligned}
$$




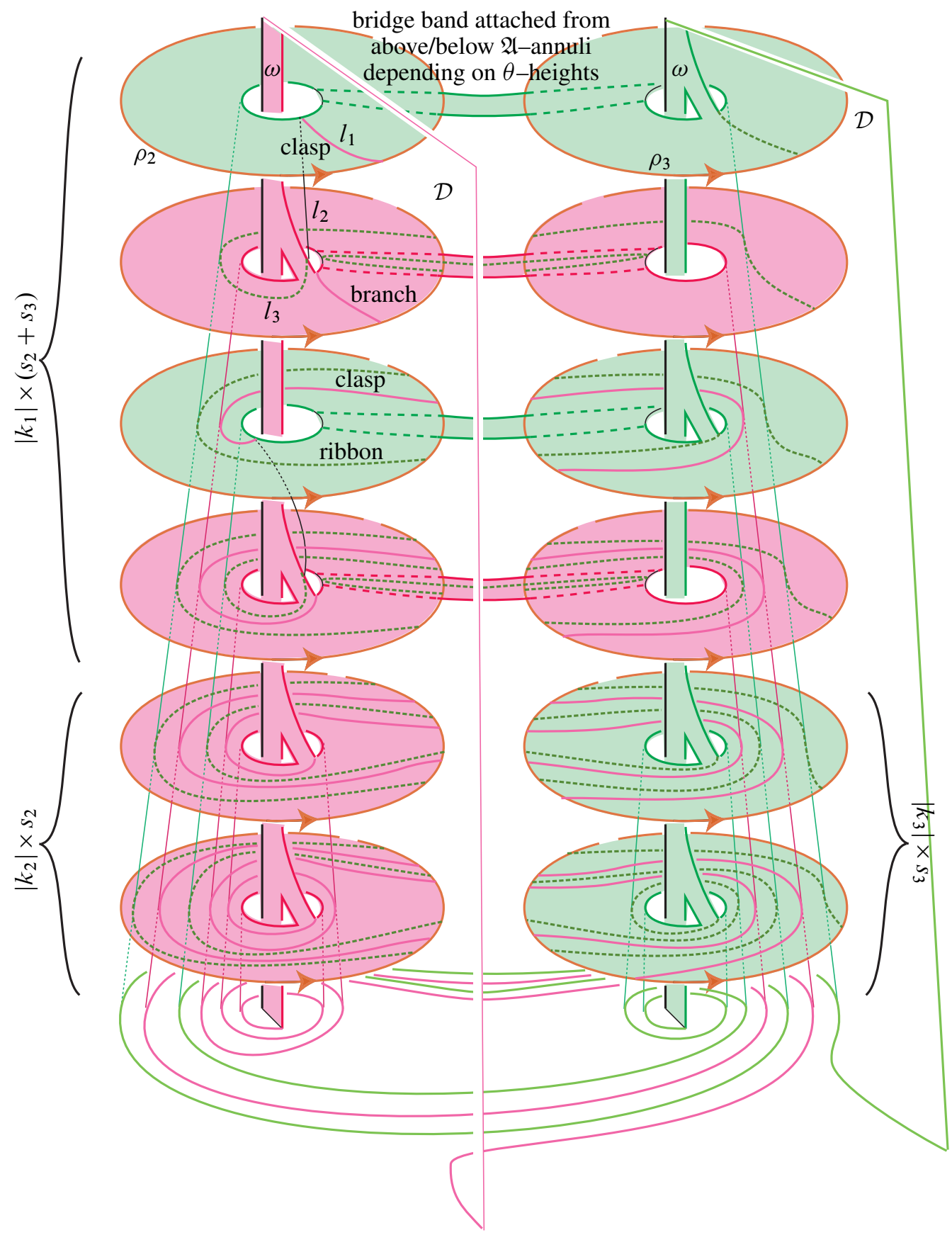

Figure 24: An immersed surface with $k_{1}=k_{2}=k_{3}=2$ and $s_{2}=s_{3}=1$ 


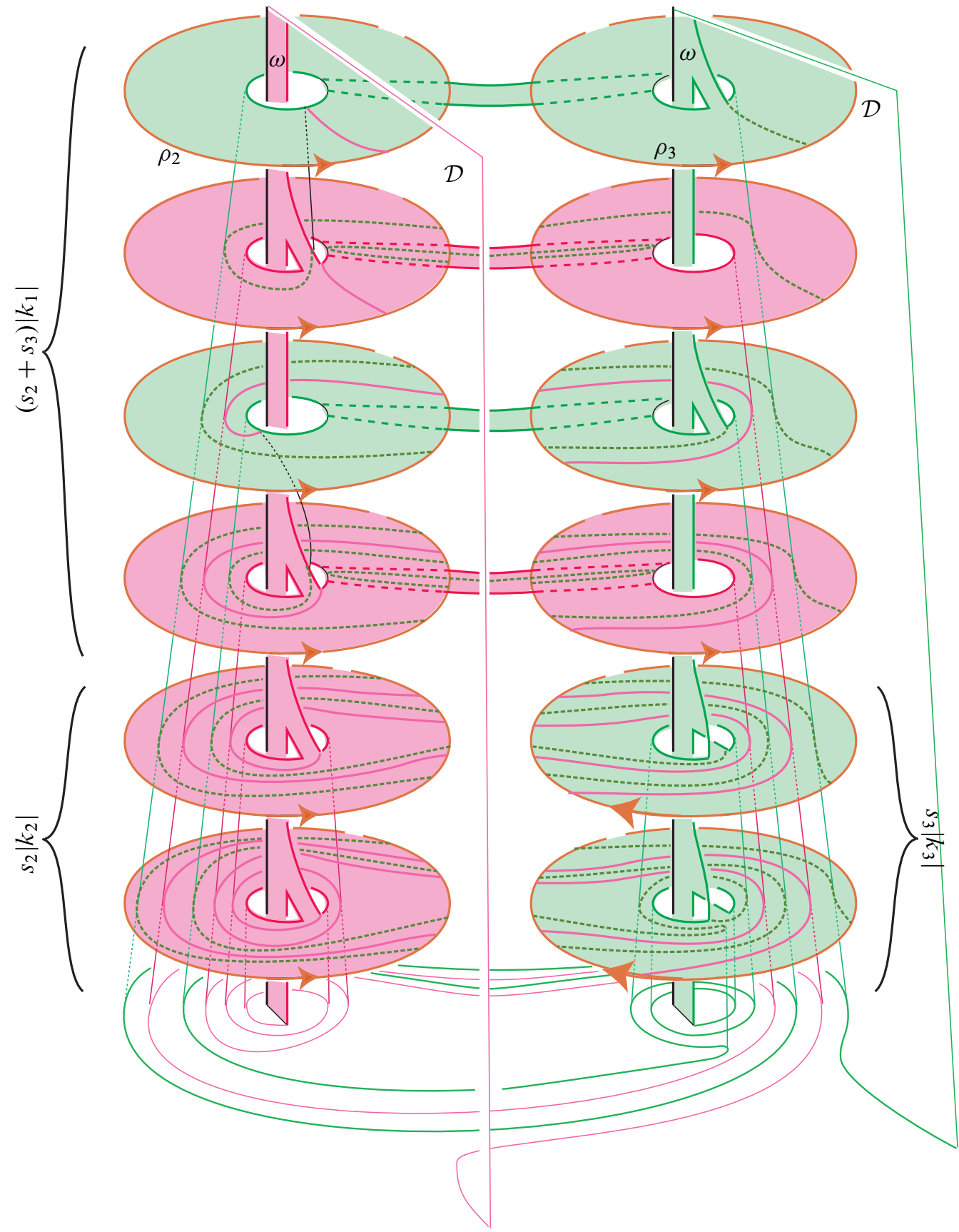

Figure 25: An immersed surface with $k_{1}=k_{2}=2, k_{3}=-2, s_{2}=s_{3}=1$ 
Compare Figure 24, where $k_{i}$ 's have the same sign, and Figure 25, where $k_{1}, k_{2}>0$ and $k_{3}<0$. Their right bottom parts are different.

Next step is to construct an immersed surface $\widehat{F}_{b}$. If $k_{1}=k_{2}=k_{3}=0$ we define $\widehat{F}_{b}=\widetilde{F}_{b}$. Otherwise apply the following:

- If $k_{1}=0$ we skip this step.

If $k_{1} \neq 0$, join the top two $\mathfrak{A}$-annuli around $\gamma_{2}$ and $\gamma_{3}$ by a band, called a bridge band as in Figures 26, 24. The bridge band connects the $\beta_{2}$ circle and the $\beta_{3}$ circle of $\widetilde{F}_{b}$. If the $\theta$-coordinate (height) of the $\mathfrak{A}$-annulus around $\gamma_{2}$ is larger (resp. smaller) than the one around $\gamma_{3}$, then the bridge band is attached to $\beta_{2}$ from below (resp. above) and to $\beta_{3}$ from above (resp. below). As a consequence, the bridge band does not tangent to the pages of the open book.

Repeat this for the first $\left(s_{2}+s_{3}\right)\left|k_{1}\right|$ pairs of $\mathfrak{A}$-annuli from the top.

- If $k_{1}=k_{2}=0$ we skip this step.

Otherwise, put $s_{2}$ copies of the $\omega$-disk, $\omega_{1}, \ldots, \omega_{s_{2}}$, about $\gamma_{2}$ (dark pink in Figure 24). Denote the $\mathfrak{A}$-annuli around $\gamma_{2}$ from the top by $\mathfrak{A}_{1}, \ldots, \mathfrak{A}_{\left|k_{1}\right|\left(s_{2}+s_{3}\right)+\left|k_{2}\right| s_{2}}$. Connect the disk $\omega_{i}$ with $\left|k_{1}\right|+\left|k_{2}\right|$ copies of $\mathfrak{A}$-annulus:

$$
\begin{aligned}
& \mathfrak{A}_{s_{3}+i}, \mathfrak{A}_{2 s_{3}+s_{2}+i}, \ldots, \mathfrak{A}_{\left|k_{1}\right| s_{3}+\left(\left|k_{1}\right|-1\right) s_{2}+i}, \\
& \mathfrak{A}_{\left|k_{1}\right|\left(s_{3}+s_{2}\right)+i}, \mathfrak{A}_{\left|k_{1}\right|\left(s_{3}+s_{2}\right)+s_{2}+i}, \ldots, \mathfrak{A}_{\left|k_{1}\right|\left(s_{3}+s_{2}\right)+\left(\left|k_{2}\right|-1\right) s_{2}+i},
\end{aligned}
$$

(when $k_{1}=0, k_{2} \neq 0$, they are $\mathfrak{A}_{i}, \mathfrak{A}_{s_{2}+i}, \ldots, \mathfrak{A}_{\left(\left|k_{2}\right|-1\right) s_{2}+i}$,

when $k_{1} \neq 0, k_{2}=0$, they are $\left.\mathfrak{A}_{s_{3}+i}, \mathfrak{A}_{2 s_{3}+s_{2}+i}, \ldots, \mathfrak{A}_{\left|k_{1}\right| s_{3}+\left(\left|k_{1}\right|-1\right) s_{2}+i}\right)$

by using the twisted bands. Depending on the signs of $k_{1}$ and $k_{2}$, the twisted band is attached differently as described in Figure 12.

- Attach $s_{2}$ copies of $\mathcal{D}$-disk about $\gamma_{1}$ to the $\mathfrak{A}$-annuli around $\gamma_{2}$.

- Similarly, attach $s_{3}$ copies of $\omega$-disk (lighter shaded in Figure 24) about $\gamma_{3}$, add $\left|k_{1}\right|+\left|k_{3}\right|$ copies of twisted bands, and $s_{3}$ copies of $\mathcal{D}$-disk.

Finally we have obtained an immersed surface $\widehat{F}_{b}$ with boundary $b$.

The following two lemmas investigate singularities of the characteristic foliation.

Lemma 5.7 If $k_{1}>0$ (resp. $\left.k_{1}<0\right)$ then the characteristic foliation for each bridge band has a single negative (resp. positive) hyperbolic singularity. See Figure 26. 


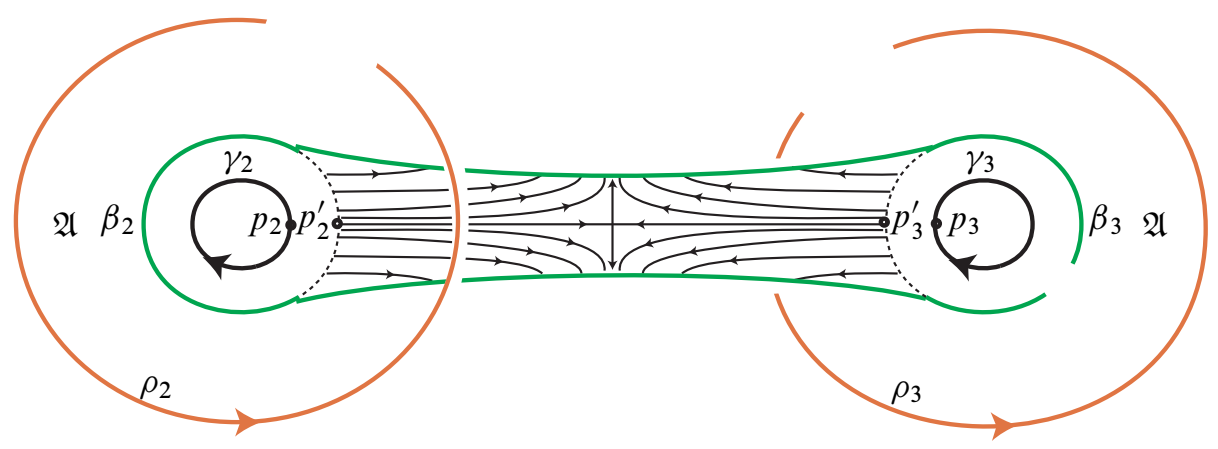

Figure 26: Characteristic foliation on a bridge band when $k_{1}>0$

Proof Let $p_{i}(i=2,3)$ be a point on the binding $\gamma_{i}$. Assume $p_{i}^{\prime} \in \beta_{i}$ is a point close to $p_{i}$ and the bridge band connects $p_{2}^{\prime}$ and $p_{3}^{\prime}$; see Figure 26. At $p_{i}$, the contact plane intersects $\gamma_{i}$ positively. Along the line segment from $p_{2}^{\prime}$ to $p_{3}^{\prime}$, the contact planes rotate $(180-\epsilon)^{\circ}$ counterclockwise. The contact plane is tangent to the bridge band at a single point somewhere between $p_{2}^{\prime}$ and $p_{3}^{\prime}$, where the hyperbolic singularity occurs. If $k_{1}>0$ (resp. $k_{1}<0$ ), the negative (positive) side of the band is facing up to the reader, thus the sign of the hyperbolic point is negative (positive).

Lemma 5.8 All the singularities of the characteristic foliation for $\left(\widehat{F}_{b} \backslash \widetilde{F}_{b}\right)$, the union of $\omega$-disks, twisted bands and $\mathcal{D}$-disks, are elliptic. Moreover, the algebraic count $e^{+}-e^{-}$of elliptic singularities for the $\omega$-disks (resp. $\mathcal{D}$-disks) is $s_{2}+s_{3}$ (resp. $\left.-s_{2}-s_{3}\right)$.

Proof As seen in the proof of Lemma 3.2, there are no hyperbolic singularities on the twisted bands.

We continue the proof of Theorem 5.6.

Figure 24 exhibits branch, clasp and ribbon intersections of $\hat{F}_{b}$. For example, in Figure 24, the union of arcs $l_{1} \cup l_{2} \cup l_{3}$ is a clasp intersection. Each pair among the $s_{2}+s_{3} \mathcal{D}$-disks about $\gamma_{1}$ gives rise to $\left|k_{1}\right|$ clasp intersections. Additionally, each pair among the $s_{2} \mathcal{D}$-disks about $\gamma_{1}$ attached to curves near $\gamma_{2}$ gives rise to $\left|k_{2}\right|$ clasp intersections, and each pair among the $s_{3} \mathcal{D}$-disks about $\gamma_{1}$ attached to curves near $\gamma_{3}$ gives rise to $\left|k_{3}\right|$ clasp intersections. In total, there are

$$
\begin{aligned}
\left|k_{1}\right|\left(\begin{array}{c}
s_{2}+s_{3} \\
2
\end{array}\right)+\left|k_{2}\right|\left(\begin{array}{c}
s_{2} \\
2
\end{array}\right)+\mid & k_{3} \mid\left(\begin{array}{c}
s_{3} \\
2
\end{array}\right) \\
& =\left(\begin{array}{c}
s_{2} \\
2
\end{array}\right)\left(\left|k_{1}\right|+\left|k_{2}\right|\right)+\left(\begin{array}{c}
s_{3} \\
2
\end{array}\right)\left(\left|k_{1}\right|+\left|k_{3}\right|\right)+s_{2} s_{3}\left|k_{1}\right|
\end{aligned}
$$


clasp intersections. Signs are assigned to each intersection according to Definition 3.10. If we count them algebraically,

(5-4) algebraic number of branches $=-s_{2}\left(k_{1}+k_{2}\right)-s_{3}\left(k_{1}+k_{3}\right)$,

$$
\text { algebraic number of clasps }=-\left(\begin{array}{c}
s_{2} \\
2
\end{array}\right)\left(k_{1}+k_{2}\right)-\left(\begin{array}{c}
s_{3} \\
2
\end{array}\right)\left(k_{1}+k_{3}\right)-s_{2} s_{3} k_{1} .
$$

Resolving all the intersection arcs, we obtain an embedded surface $\Sigma_{b}$.

By Proposition 3.8 and Corollary 3.9, the resolution of branch, clasp and ribbon intersections create additional hyperbolic singularities. The total algebraically counted number of such hyperbolic points is

$$
(5-4)+2 \times(5-5)=-\left\{\left(s_{2}+s_{3}\right)^{2} k_{1}+s_{2}^{2} k_{2}+s_{3}^{2} k_{3}\right\} .
$$

In summary we have for elliptic singularities

$$
\begin{aligned}
& e^{+}=(n ; \delta \text {-disks })+\left(s_{2}+s_{3} ; \omega \text {-disks }\right), \\
& e^{-}=\left(s_{2}+s_{3} ; \mathcal{D} \text {-disks }\right),
\end{aligned}
$$

and for hyperbolic singularities

$h^{+}-h^{-}$

$=\left(a_{\sigma}\right.$; bands between $\delta$-disks $)+\left(a_{\rho_{2}}+a_{\rho_{3}}\right.$; bands between $\delta_{n}$ and $\mathfrak{A}$-annuli $)$

$-\left(k_{1}\left(s_{2}+s_{3}\right)\right.$; bridge bands; Lemma 5.7)

$-\left(\left(s_{2}+s_{3}\right)^{2} k_{1}+s_{2}^{2} k_{2}+s_{3}^{2} k_{3} ;\right.$ resolution of branches, clasps, ribbons (5-6))

$\stackrel{(5-2)}{=} a_{\sigma}+a_{\rho_{2}}+a_{\rho_{3}}-s_{2}\left(a_{\rho_{2}}+k_{1}\right)-s_{3}\left(a_{\rho_{3}}+k_{1}\right)$

$=a_{\sigma}+a_{\rho_{2}}\left(1-s_{2}\right)+a_{\rho_{3}}\left(1-s_{3}\right)-\left(s_{2}+s_{3}\right) k_{1}$.

Finally we have

$$
\begin{aligned}
\operatorname{sl}\left(b,\left[\Sigma_{b}\right]\right) & =-\left(e^{+}-e^{-}\right)+\left(h^{+}-h^{-}\right) \\
& =-n+a_{\sigma}+a_{\rho_{2}}\left(1-s_{2}\right)+a_{\rho_{3}}\left(1-s_{3}\right)-\left(s_{2}+s_{3}\right) k_{1} .
\end{aligned}
$$

\section{References}

[1] J W Alexander, A lemma on systems of knotted curves, Proc. Natl. Acad. Sci. USA 9 (1923) 93-95

[2] D Bennequin, Entrelacements et équations de Pfaff, from: "Third Schnepfenried geometry conference, Vol. 1 (Schnepfenried, 1982)", Astérisque 107, Soc. Math. France (1983) 87-161 MR753131 
[3] J S Birman, Braids, links, and mapping class groups, Annals of Math. Studies 82, Princeton Univ. Press (1974) MR0375281

[4] Y Eliashberg, Contact 3-manifolds twenty years since J Martinet's work, Ann. Inst. Fourier (Grenoble) 42 (1992) 165-192 MR1162559

[5] J B Etnyre, Transversal torus knots, Geom. Topol. 3 (1999) 253-268 MR1714912

[6] J B Etnyre, Legendrian and transversal knots, from: "Handbook of knot theory", (W Menasco, M Thistlethwaite, editors), Elsevier, Amsterdam (2005) 105-185 MR2179261

[7] J B Etnyre, K Honda, On symplectic cobordisms, Math. Ann. 323 (2002) 31-39 MR1906906

[8] J B Etnyre, B Ozbagci, Invariants of contact structures from open books, Trans. Amer. Math. Soc. 360 (2008) 3133-3151 MR2379791

[9] B Farb, D Margalit, A primer on mapping class groups, version 5.0, Preprint (2011) Available at http://www.math.uchicago.edu/ margalit/mcg/mcgv50.pdf

[10] H Geiges, An introduction to contact topology, Cambridge Studies in Advanced Math. 109, Cambridge Univ. Press (2008) MR2397738

[11] E Giroux, Géométrie de contact: de la dimension trois vers les dimensions supérieures, from: "Proceedings of the International Congress of Mathematicians, Vol. II (Beijing, 2002)", (T Li, editor), Higher Ed. Press, Beijing (2002) 405-414 MR1957051

[12] N Goodman, Overtwisted open books from sobering arcs, Algebr. Geom. Topol. 5 (2005) 1173-1195 MR2171807

[13] K Honda, On the classification of tight contact structures. I, Geom. Topol. 4 (2000) 309-368 MR1786111

[14] B Ozbagci, A I Stipsicz, Surgery on contact 3-manifolds and Stein surfaces, Bolyai Society Math. Studies 13, Springer, Berlin (2004) MR2114165

[15] E Pavelescu, Braids and open book decompositions, $\mathrm{PhD}$ thesis, University of Pennsylvania (2008) Available at http://www.math.upenn.edu/grad/dissertations/ ElenaPavelescuThesis.pdf

Department of Mathematics, University of Iowa

14 MacLean Hall, Iowa City IA 52242, USA

Department of Mathematics, Rice University

6100 Main St, Houston, TX 77005, USA

kawamuro@iowa.uiowa.edu, elena.pavelescu@rice.edu

Received: 8 November $2009 \quad$ Revised: 6 October 2010 